\title{
Formulation of reference solutions for compaction process in sedimentary basins
}

\author{
Paulo Sérgio B. Lemos ${ }^{1}$ ๑ \\ ${ }^{1}$ Department of Civil Engineering, Federal \\ University of Rio Grande do Sul, Porto \\ Alegre, Brazil \\ ${ }^{2}$ Geosciences Division, IFP Energies \\ nouvelles, 1 et 4 avenue de Bois-Préau, \\ Rueil-Malmaison, 92852, France
}

\section{Correspondence}

Paulo Sérgio B. Lemos, Department of Civil Engineering, Federal University of Rio Grande do Sul, Porto Alegre, Brazil. Email: paulobaumbach@hotmail.com

\author{
André Brüch $^{2}$ (i) | Samir Maghous ${ }^{1}$ (
}

\begin{abstract}
Summary
This paper is devoted to the development of semianalytical solutions for the deformation induced by gravitational compaction in sedimentary basins. Formulated within the framework of coupled plasticity-viscoplasticity at large strains, the modeling dedicates special emphasis to the effects of material densification associated with large irreversible porosity changes on the stiffness and hardening of the sediment material. At material level, the purely mechanical compaction taking place in the upper layers of the basin is handled in the context of finite elastoplasticity, whereas the viscoplastic component of behavior is intended to address creep-like deformation resulting from chemomechanical that prevails at deeper layers. Semianalytical solutions describing the evolution of mechanical state of the sedimentary basin along both the accretion and postaccretion periods are presented in the simplified oedometric setting. These solutions can be viewed as reference solutions for verification and benchmarks of basin simulators. The proposed approach may reveal suitable for parametric analyses because it requires only standard mathematics-based software for PDE system resolution. The numerical illustrations provide a quantitative comparison between the derived solutions and finite element predictions from an appropriate basin simulator, thus showing the ability of the approach to accurately capture essential features of basin deformation.
\end{abstract}

\section{KE Y W O R D S}

coupled plasticity-viscoplasticity, gravitational compaction, hardening, large strains, sedimentary basin

\section{1 | INTRODUCTION}

Sedimentary basins are natural structures with great economical interest owing to hydrocarbons, groundwater, and mineral reserves. Assessment and exploration of these resources require a comprehensive understanding of the multiple coupled phenomena that occur over geological timescale, and for this purpose, analytical and numerical models are of great importance as they allow to test different scenarios of a basin history.

One of the key aspects of basin simulation concerns the mechanical model used to describe compaction through time as tectonic subsidence and basin deformation are strongly coupled with thermal evolution and fluid flow. In this regard, the numerical model should be able to deal with the compaction mechanisms that may take place in different types of groups of sediments to appropriately reconstruct the geological events through time. 
In siliciclastic rocks, for example, two main types of compaction can take place: purely mechanical compaction due to grain rearrangement and subsequent pore fluid expulsion, and chemo-mechanical compaction resulting from dissolution, diffusion, and precipitation of minerals, known as intergranular pressure solution (IPS). The first prevails in the early stages of a newly deposited layer, whereas the second progressively dominates as continuous burial increases sediments temperature and effective stresses. ${ }^{1}$

Understanding the mechanics of deformation in sedimentary basins has been the subject of study for a long time. In 1930, the geophysicist Lawrence F. Athy ${ }^{2}$ published a pioneering contribution to the modeling of sediments compaction process. It basically consists of an empirical law that describes rock porosity reduction with burial depth as an exponential decay. Relying upon phenomenological relationships that relates porosity to rock overburden, the concept of porosity versus Terzaghi's effective vertical stress dependence has been then introduced by Hubbert and Rubey ${ }^{3}$ and later by Smith. ${ }^{4}$ In this framework, the empirical porosity-stress law must be calibrated for each specific rock according to available well data.

These ideas have been widely adopted and implemented in numerical finite element (FE) models and are still applied in Basin and Petroleum System Modeling (BPSM) to represent mechanical compaction under the assumption of oedometric evolution. ${ }^{5,6}$ However, such models are not devised for relevant assessment of the horizontal stresses induced by compaction phenomena, nor for capturing the impact of lateral deformations induced by tectonics, which may strongly affect the poro-mechanical state of the basin, eventually resulting in seal rock fracturing and fault reactivation. To overcome this limitation inherent to the analyses based on phenomenological porosity-stress laws, several contributions have focused in the last decades on the formulation of constitutive models that rely upon a more comprehensive description of the mechanics involved in basin deformation. ${ }^{7-16}$ Developed in the context of tensorial formalism, these models have been applied in basin simulations under different geological scenarios, demonstrating the importance of addressing deformation in sedimentary basins within a three-dimensional (3D) framework. As regards the chemical fluid-rock interactions and their implications on the sedimentary material behavior, several approaches have been devoted to describe the complex aspects of dissolution and precipitation of minerals in siliciclastic and carbonate rocks. ${ }^{17-26}$ Still, the formulation of a macroscopic material model that accounts for chemical diagenetic processes in basin modeling remains a challenging issue. In addition, factors such as the presence of hydrocarbons or grain coating may retard or totally inhibit pressure-solution even at great depths. ${ }^{27-30}$ Despite these difficulties, different approaches aimed at addressing chemo-mechanical compaction in sedimentary basins at macroscopic level have been formulated in literature. ${ }^{5,31-35}$

To deal with these coupled phenomena, Brüch et al. ${ }^{36}$ developed a constitutive model for the fully saturated porous material in a thermo-poro-mechanics framework, which was incorporated in a numerical tool on the basis of the FE method. This model has its origins in previous works, ${ }^{37-44}$ which aimed to describe purely mechanical compaction of sediments. Based on micromechanical reasoning, the formulation takes into account the effects of large irreversible strains on the poroelastic properties of the basin through additional terms in the state equations of the porous material. This is relevant in basin simulation as some sediment layers may be subjected to more than $50 \%$ of porosity reduction. $^{45}$

Nevertheless, the accuracy of numerical simulators must be verified for each engineering application by comparing the predicted results to available benchmarks and reference solutions. In that respect, one may quote the numerical and analytical solutions via Fourier transforms to classical geomechanics problems formulated within the framework of coupled thermo-poro-mechanics. ${ }^{46-50}$ The analytical solutions are derived in the context of both infinitesimal ${ }^{46}$ and large strains $s^{48,49}$ by resorting to Laplace transform and Fourier series to solve the resulting boundary value problems (BVPs). This work presents the formulation of analytical and semianalytical reference solutions that describe the deformation processes in a sedimentary basin. These reference solutions can be conveniently used for verification and validation benchmarks of numerical basin simulators. The mechanical and chemo-mechanical compaction processes are respectively represented by plastic and viscoplastic models in the context of large irreversible strains. The analysis is restricted to drained and isothermal conditions, disregarding the effect of pore pressure and temperature on the material. The semianalytical solutions are compared with those obtained by the numerical simulator developed by Brüch et al. ${ }^{36}$

It should be noted that the proposed analysis is developed within a purely academic situation that relies upon a simplified geological scenario for compaction processes. In that respect, the primary objective of the paper is to provide reference solutions derived for compaction in sedimentary basins in the context of highly simplified setting, and not to predict the mechanical state that would prevail in real data life basin. 


\section{2 | STATEMENT OF THE PROBLEM}

The mechanical problem under consideration refers to the evaluation of stresses and strains developing in a sedimentary basin under oedometric conditions during both the formation phases by continuous accretion of sediment material and along the postaccretion phase as well. The analysis shall focus on deformation induced in the basin by purely mechanical and chemo-mechanical compaction processes. Whereas purely mechanical compaction originates mainly from rearrangement of the solid particles during burial and can thus be modeled in the framework of plasticity, chemomechanical compaction resulting from IPS phenomena is generally associated with creep-like deformation. In addition, compaction process in a sedimentary basin generally involves large strains, the reduction in porosity of the sediment material exceeding in many situations values as high $^{45}$ as $50 \%$. The theoretical framework of coupled elastoplasticityviscoplasticity at finite strains appears therefore suitable for accurate description of the mechanics controlling the basin deformation.

In order to formulate semianalytical solutions for the compaction process in sedimentary basins, a simplified setting relying upon the following assumptions is adopted: (a) the whole sedimentation process takes place under oedometric conditions; (b) the sediment constitutive material exhibits homogeneous and isotropic mechanical properties in its reference state, that is, at the time it is deposited at the top of the basin; (c) the hydromechanical coupling is disregarded in the subsequent analysis (i.e., the effect of pore pressure is not considered), which amounts to addressing the particular case of highly permeable sediment material (fully drained conditions); and (d) the mechanical evolution of the basin is analyzed under isothermal condition, disregarding the effect of geothermal gradient on the material properties and deformation. The role of pore pressure and geothermal gradient has been assessed through numerical formulations in previous works. ${ }^{9,41,44,51,52}$

The sedimentary basin undergoing compaction is modeled as an infinite layer, perpendicular to the $\underline{e}_{3}$ direction and lying on a rigid substratum along the plane $x_{3}=0$. Neglecting the tectonic activity, the gravitational field $\underline{g}=-g \underline{e}_{3}$ stands for the only external loading in the compaction process. In addition, the anisotropy of constitutive properties of the material in its reference state and that induced by compaction processes are neglected in the analysis. This framework of assumptions allows for simplified description of the problem geometry and geological process as well as for more analytically tractable field equations governing the evolution of mechanical state of the sedimentary basin. In this simplified framework, the physical quantities involved in the problem only depend on time and the vertical coordinate $x_{3}$. The position of a material particle in the sedimentary layer at a time $t$ is defined by coordinate as $x_{3}$, whereas the instant when the particle is deposited at the top of the layer is referred to as $T\left(x_{3}, t\right)$.

As the sediments are continuously deposited at the top of the basin, the sediment layer thickness is time dependent. Assuming that the top of the layer remains horizontal, the position of the upper boundary is defined by the gravitational compaction law $x_{3}=H(t)$ (Figure 1$)$.

\section{1 | Governing field equations}

The quasistatic BVP is defined by two field equations, which are briefly described in the sequel. Neglecting the inertial effects, the momentum balance equation for the continuum reads as follows:

FI G URE 1 Geometry model for sedimentary basin and loading conditions

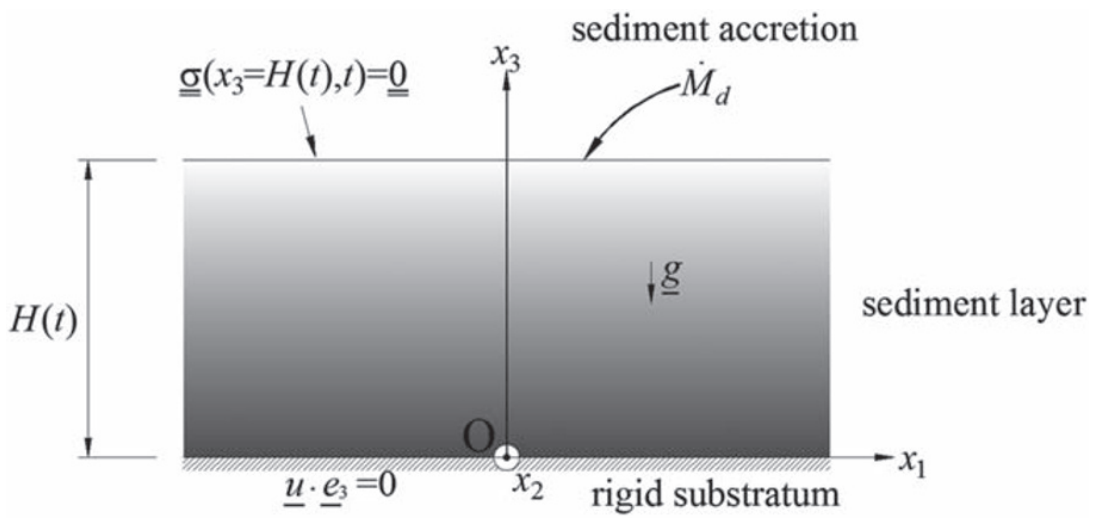




$$
\operatorname{div} \underline{\underline{\sigma}}\left(x_{3}, t\right)+\rho\left(x_{3}, t\right) \underline{g}=\underline{0},
$$

where $\underline{\underline{\sigma}}$ is the Cauchy stress tensor and $\rho$ is the mass density of the sediment material.

In the Eulerian formulation, the mass balance equation writes as follows:

$$
\frac{\partial \rho\left(x_{3}, t\right)}{\partial t}+\operatorname{div}\left(\rho\left(x_{3}, t\right) \underline{u}\left(x_{3}, t\right)\right)=0
$$

where $\underline{u}$ is the Eulerian velocity field of the sediment particles.

The Lagrangian counterpart of the mass balance equation is

$$
\rho\left(x_{3}, t\right)=\frac{\rho_{0}}{J\left(x_{3}, t\right)},
$$

where $\rho_{0}$ is the initial mass density and $J=d \Omega_{t} / d \Omega_{0}$ is the Jacobian of the transformation, that is, the ratio of the volume of a particle at the current configuration to the initial configuration.

Physical quantities associated with the particles seated at the top of the sedimentary basin, viewed as an open material system, comply with the following initial conditions:

$$
\begin{aligned}
& \underline{\underline{\sigma}}(H(t), t)=\underline{\underline{0}}, \\
& J(H(t), t)=1, \\
& \rho(H(t), t)=\rho_{0} .
\end{aligned}
$$

As a boundary condition, the velocity of the particles in contact with the rigid substratum is null

$$
\underline{u}(0, t) \cdot \underline{e}_{3}=0 \text {. }
$$

In the context of oedometric compaction setting, which implicitly disregards the effects of both tectonic events and loading induced anisotropy, together with assumption of homogeneity of the deposited sediment material along the whole accretion phase, the general form for velocity and stress fields express as

$$
\begin{gathered}
\underline{u}\left(x_{3}, t\right)=u_{3}\left(x_{3}, t\right) \underline{e}_{3}, \\
\underline{\underline{\sigma}}\left(x_{3}, t\right)=\sigma_{h}\left(x_{3}, t\right)\left(\underline{e}_{1} \otimes \underline{e}_{1}+\underline{e}_{2} \otimes \underline{e}_{2}\right)+\sigma_{v}\left(x_{3}, t\right) \underline{e}_{3} \otimes \underline{e}_{3} .
\end{gathered}
$$

Accordingly, the deviatoric part of stress tensor reads:

$$
\underline{\underline{s}}=\underline{\underline{\sigma}}-\frac{1}{3} \operatorname{tr} \underline{\underline{\sigma 1}}=\frac{1}{3}\left(\sigma_{\nu}-\sigma_{h}\right)\left(-\underline{e}_{1} \otimes \underline{e}_{1}-\underline{e}_{2} \otimes \underline{e}_{2}+2 \underline{e}_{3} \otimes \underline{e}_{3}\right)
$$

\subsection{Loading and geometrical transformation}

The mass of sediments deposited per unit area $M_{d}(t)$ on the top of the basin during the time interval $[0, t]$ characterizes the magnitude of the loading applied to the sedimentary basin. It expresses as 


$$
M_{d}(t)=\int_{0}^{H(t)} \rho\left(x_{3}, t\right) d x_{3} .
$$

It is assumed that the rate of sediments supply $\dot{M}_{d}(t)$ is prescribed. The rate-form of 11 writes

$$
\dot{M}_{d}(t)=\dot{H}(t) \rho_{0}+\int_{0}^{H(t)} \frac{\partial \rho\left(x_{3}, t\right)}{\partial t} d x_{3} .
$$

The combination of 2 and 12 together with the boundary condition 7 leads to

$$
\dot{H}(t)=\frac{\dot{M}_{d}(t)}{\rho_{0}}+u_{3}(H(t), t) .
$$

The ratio between the height of a particle in the current configuration at time $t$ and its height in the reference state at time $T\left(x_{3}, t\right)$ defines by the vertical stretch $\Lambda\left(x_{3}, t\right)$ (Figure 2). Based on its definition, the initial condition for the sediment particle is

$$
\Lambda(H(t), t)=1
$$

Under oedometric conditions, the gradient of the geometrical transformation of a particle between the reference and the current states takes the following form:

$$
\underline{\underline{F}}\left(x_{3}, t\right)=\underline{e}_{1} \otimes \underline{e}_{1}+\underline{e}_{2} \otimes \underline{e}_{2}+\Lambda\left(x_{3}, t\right) \underline{e}_{3} \otimes \underline{e}_{3} .
$$

The Jacobian of the geometrical transformation $J=\operatorname{det} \underline{\underline{F}}$ is therefore equal to the vertical stretch:

$$
J=\operatorname{det} \underline{\underline{F}}=\Lambda .
$$

Because $\underline{\underline{F}}\left(x_{3}, t\right)$ and $\Lambda\left(x_{3}, t\right)$ depend on the coordinate $x_{3}$ in the current configuration, these quantities are as Eulerian fields, even if their definition is similar to that classically adopted in the Lagrangian description of a solid transformation.

The Eulerian gradient of the velocity field $\nabla \underline{u}$ is related to $\underline{\underline{F}}$ according to $\nabla \underline{u}=\underline{\underline{\underline{F}}} \cdot \underline{\underline{F}}{ }^{-1}$, which leads to

$$
\frac{\partial u_{3}}{\partial x_{3}} \underline{e}_{3} \otimes \underline{e}_{3}=\frac{\dot{\Lambda}}{\Lambda} \underline{e}_{3} \otimes \underline{e}_{3} \rightarrow \frac{\partial u_{3}}{\partial x_{3}}=\frac{\dot{\Lambda}}{\Lambda}
$$

The strain rate tensor $\underline{\underline{d}}=\frac{1}{2}\left(\nabla \underline{u}+{ }^{t} \nabla \underline{u}\right)$ is

F I G U RE 2 Definition of the particle vertical stretch

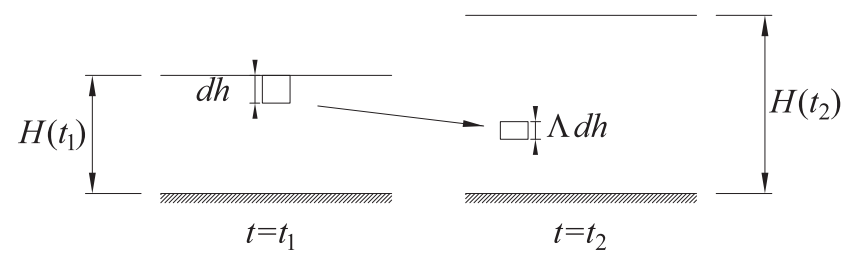




$$
\underline{\underline{d}}=\frac{\partial u_{3}}{\partial x_{3}} \underline{e}_{3} \otimes \underline{e}_{3}=\frac{\dot{\Lambda}}{\Lambda} \underline{e}_{3} \otimes \underline{e}_{3} .
$$

From equilibrium arguments, the vertical component of stress at depth $x_{3}$ can be evaluated as the total weight of the material volume having unit cross-section area that is seated above the considered depth:

$$
\sigma_{v}\left(x_{3}, t\right)=-\int_{x_{3}}^{H(t)} \rho(\eta, t) g d \eta
$$

The rate of vertical stress can be calculated from 19 by making use of 2 and 13, which results in

$$
\dot{\sigma}_{v}\left(x_{3}, t\right)=-\dot{M}_{d}(t) g
$$

\section{3 | Constitutive behavior of sediment material}

The sedimentary material is modeled as an isotropic elastic-plastic-viscoplastic material undergoing large strains. As mentioned previously, the anisotropy induced by the compaction on the sediment mechanical properties is disregarded. During the geometric transformation, the reversible strains (elastic) are assumed to remain infinitesimal. Large strains produced by compaction are therefore of irreversible nature.

The solid phase that constitutes the skeleton particle is considered to be incompressible. The solid mass balance implies therefore that the Eulerian porosity (current pore volume fraction) expresses as ${ }^{40}$

$$
\varphi\left(x_{3}, t\right)=1-\frac{1-\phi_{0}}{J\left(x_{3}, t\right)} \approx 1-\frac{1-\phi_{0}}{J_{i r}\left(x_{3}, t\right)},
$$

where $\phi_{0}=\varphi(H(t), t)$ refers to sediment porosity in the reference state and $J_{i r}$ is the irreversible component of the Jacobian transformation, which is close to the total Jacobian $J_{i r} \approx J$ owing to the assumption of infinitesimal reversible strains.

The previous equation relates current porosity to volumetric dilatation of the sediment material during burial. It is expected that the large porosity variation modifies the material elastic properties. ${ }^{40}$ The stiffness increase of the skeleton elastic modulus induced by the progressive decrease in porosity is modeled by the Hashin-Shtrikman upper bounds formulated for isotropic composite materials. These variational bounds coincide with the micromechanical estimates derived from Mori-Tanaka scheme, ${ }^{53}$ which are known to reasonably model the elastic properties of isotropic porous media. ${ }^{54,55}$ The expressions for the bulk and shear moduli as a function of porosity are given by

$$
\left\{\begin{array}{l}
K(\varphi)=\frac{4 k^{s} \mu^{s}(1-\varphi)}{3 k^{s} \varphi+4 \mu^{s}} \\
\mu(\varphi)=\frac{\mu^{s}(1-\varphi)\left(9 k^{s}+8 \mu^{s}\right)}{k^{S}(9+6 \varphi)+\mu^{s}(8+12 \varphi)}
\end{array}\right.
$$

where $k^{s}$ and $\mu^{s}$ are the bulk and shear moduli of the solid phase, which are assumed to be unaffected by compaction processes.

Before further developments, it should be emphasized that the ability of such a formulation to provide relevant elastic estimates over a wide range of rocks and porosity remains to be assessed through specific experimental tests. Keeping in mind, however, that the objective herein is not to represent a specific type of rock but rather to model in a consistent framework the stiffness increase associated with material compaction, these micromechanics-based estimates will be adopted in a first approach with the aim to qualitatively reproduce this feature. 
Equation 22 together with 21 introduces a strong coupling between elasticity and plastic-viscoplastic component of the constitutive behavior. It is shown that the state equations describing the stress-strain relationship can be formulated in rate-form as follows ${ }^{40}$ :

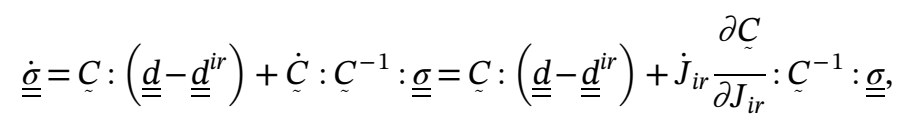

where $\underline{\underline{\dot{\sigma}}}$ is the Cauchy stress rate tensor, $\underline{\underline{d}}^{i r}$ is the irreversible part of the strain rate tensor, and the fourth-order tensor $C$ is the material elastic stiffness moduli, in which the expression under the assumption of isotropy is

$$
\underset{\sim}{C}(\varphi)=(K(\varphi)-2 \mu(\varphi) / 3) \underline{\underline{1}} \otimes \underline{\underline{1}}+2 \mu(\varphi) \underline{\sim},
$$

where 1 and 1 refer respectively to the second-order and fourth-order identity tensors.

The term $\dot{\widetilde{C}}: C^{-1}: \underline{\underline{\sigma}}$ in 23 represents the influence of large irreversible strains on elastic properties. In the context of the oedometric hypothesis, 23 does not involve terms referring to large rotations, and the related term reduces to

$$
\dot{\sim}: C^{-1}: \underline{\underline{\sigma}}=\frac{\operatorname{tr} \underline{\underline{\underline{\sigma}}}}{3} \frac{\dot{K}}{K} \underline{\underline{\underline{1}}}+\frac{\dot{\mu}}{\mu} \underline{\underline{\underline{S}}}
$$

Additionally, the relationship between the volumetric irreversible strain rate and the irreversible component of Jacobian reads

$$
\operatorname{trd}_{\underline{d^{i r}}}=\frac{\dot{J}_{i r}}{J_{i r}}
$$

\section{4 | Plastic behavior}

The irreversible strain rate is additively split into two contributions, $\underline{\underline{d}}^{i r}=\underline{\underline{d}}^{p}+\underline{\underline{d}}^{v p}$, related to the plastic and viscoplastic strain components.

The plastic component of the constitutive model aims at representing purely mechanical compaction resulting from rearrangement of the solid particle during burial. The fundamental features that should be specified for the plastic behavior are related to the plasticity yield surface and to its evolution controlled by the associated hardening law. Regarding the first aspect, we resort to the concept of so-called "cap models" for the formulation of a simplified isotropic plastic criterion. Referring to the plane $\left(p=-I_{1} / 3=-\operatorname{tr} \underline{\underline{\sigma}} / 3, q=J_{2}=\sqrt{\underline{\underline{s}}: \underline{\underline{s}} / 2}\right)$, the yield surface depicted in Figure 3 is bounded in the dilation domain by a straight line that stands for the brittle failure regime (critical line), whereas the

F I G U RE 3 Schematic representation of simplified plastic yield surface

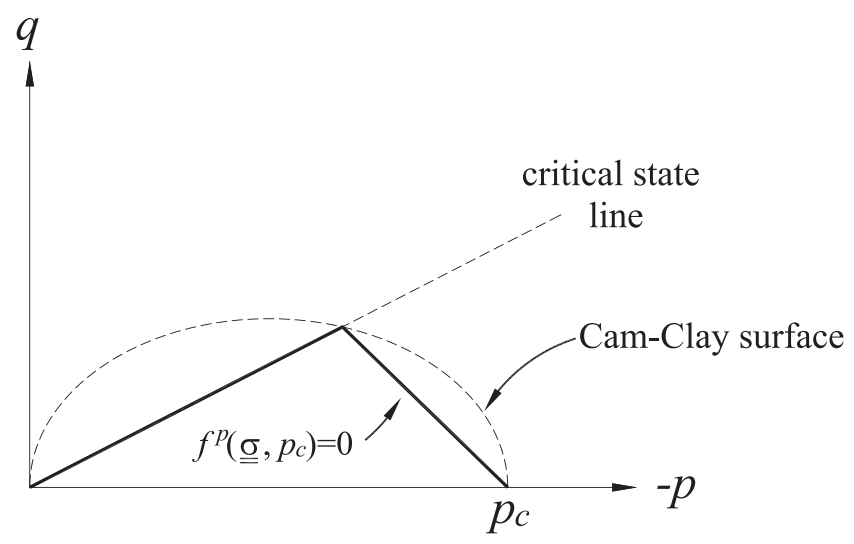


side corresponding to ductile failure and material hardening (contracting state) is also approximated in this analysis by an inclined straight line describe by the following criterion:

$$
f^{p}\left(\underline{\underline{\sigma}}, p_{c}\right)=-\frac{1}{3} \operatorname{tr} \underline{\underline{\sigma}}+a \sqrt{\frac{1}{2} \underline{\underline{s}}: \underline{\underline{s}}}-p_{c}=0
$$

where $p_{c}$ is the consolidation pressure (similar to that introduced in the Cam-Clay model) and represents the hardening parameter in the model, whereas $a$ is a positive scalar that controls the slope of the ductile part of yield surface. It is emphasized that such a simplified yield criterion has already been adopted for petroleum engineering applications. ${ }^{56}$ It is important to observe that in the absence of tectonic loading, compaction processes are expected to produce purely contracting stress states. In this context, the simplified plastic criterion 27 appears suitable for representing yielding under oedometric stress paths such as those involved within the purely gravitational compaction resulting from sediments overburden, while remaining tractable for devising analytical developments at the sedimentary basin level. In contrast, it would not be relevant for modeling complex geological scenarios involving for instance lateral shortening. In such situations, more sophisticated yield surfaces would be necessary in order to include the possibility of shearinduced dilation for both drained and undrained material behavior. ${ }^{9,14}$

An associated flow rule is adopted for the plastic strain rate:

$$
\underline{\underline{d}}^{p}=\dot{\chi} \frac{\partial f^{p}}{\partial \underline{\underline{\sigma}}}
$$

where $\dot{\chi}$ is the nonnegative plastic multiplier rate.

The plastic hardening law describes the evolution of the consolidation pressure due to the irreversible material densification. A formulation based on limit analysis and micromechanics has been originally proposed in Barthélémy et al. ${ }^{42}$ for $p_{c}$. This formulation has been recently modified by Brüch et al. ${ }^{52}$ using a calibration exponent $m_{p}$ :

$$
p_{c}(\varphi)=p_{c 0}\left(\frac{\ln \varphi}{\ln \phi_{0}}\right)^{m_{p}}
$$

where $p_{c 0}$ is the initial consolidation pressure for plasticity.

It is emphasized that his hardening law prevents the development of negative porosities under high isotropic compression, because $\lim _{\varphi \rightarrow 0} p_{c}=+\infty$, which ensures by virtue of 21 that $J_{i r}$ remains always higher than $J_{i r}^{c r}=1-\phi_{0}{ }^{43}$

The rate-form expression of the hardening law 29 expresses as follows:

$$
\dot{p}_{c}=-h_{p}(\varphi) \frac{\dot{J}_{i r}}{J_{i r}} \operatorname{with} h_{p}(\varphi)=-\frac{m_{p}\left(1-\phi_{0}\right) p_{c}}{J_{i r} \varphi \ln \varphi}
$$

where $h_{p}$ is the plastic hardening modulus.

\section{5 | Viscoplastic behavior}

The viscoplastic component of the constitutive model aims to represent the chemo-mechanical compaction induced by the IPS. The yield surface for viscoplastic component of the behavior is defined in a similar way than for plastic behavior, resorting once again to the concept of "cap models". In the range of contractive stress states relevant to oedometric compaction process, the boundary of the yield surface is described by the following:

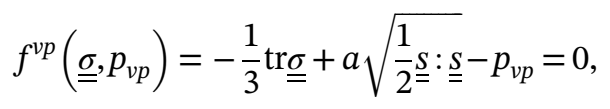

where the consolidation pressure $p_{v p}$ stands for the hardening law of the viscoplastic model. 
The time-dependent component of the strain rate is based on the generalized Perzyna's overstress theory ${ }^{57}$ :

$$
\underline{\underline{d}}^{v p}=\frac{\left\langle f^{v p}\right\rangle}{\eta_{v p}} \frac{\partial g^{v p}}{\partial \underline{\underline{\sigma}}}
$$

where $\langle\cdot\rangle$ is the Macaulay brackets, $\eta_{v p}$ is the viscosity coefficient, and $g^{v p}$ is the viscoplastic potential defining the direction of viscoplastic strain rate. An associated flow rule $g^{v p}=f^{v p}$ shall be assumed in the subsequent analysis.

The evolution law for $p_{v p}$ has been formulated ${ }^{51}$ and stems from the heuristic idea that similarity can be preserved between the plastic and viscoplastic models:

$$
p_{v p}(\varphi)=p_{v p 0}\left(\frac{\ln \varphi}{\ln \phi_{0}}\right)^{m_{v p}}
$$

where $p_{c 0}$ is the initial consolidation pressure for viscoplasticity and the exponent $m_{v p}$ is a material constant ranging between zero and unity that controls the relative magnitude of viscoplastic strains with regard to plastic strains.

The rate-form expression of the hardening law for $p_{v p}$ is given by

$$
\dot{p}_{v p}=-h_{v p}(\varphi) \frac{\dot{J}_{i r}}{J_{i r}} \text { with } h_{v p}(\varphi)=-\frac{m_{v p}\left(1-\phi_{0}\right) p_{v p}}{J_{i r} \varphi \ln \varphi}
$$

where $h_{v p}$ is the viscoplastic hardening modulus.

In order to reproduce the observations from real data life basins, the initial value of viscoplastic consolidation pressure should be higher than that the plastic counterpart; that is, $p_{c 0}<p_{v p 0}$. This condition, assumed throughout the analysis, simply expresses that purely mechanical compaction prevails in the upper layers of the sedimentary basin. In other words, plastic strains are first activated during burial and at each instant so that the plastic layers are located above the viscoplastic layers.

\section{3 | MECHANICAL FORMULATION OF THE PROBLEM}

The main objective of this section is to formulate solutions that describe the mechanical behavior of the sedimentary basin. For this purpose, a description of the problem is initially presented.

The geological time evolution of the sedimentary basin under compaction processes is divided into five consecutive phases (Figure 4), distinct from each other by the behavior ranges involved along the basin layers. The occurrence of each phase is conditioned by the whole basin data considered for the analysis.

Referring to Figure 4, the first four phases refer to the sediment deposition period (basin formation), whereas the latter one refers to the postdepositional period.

- Elastic phase: This phase marks the beginning of the sediment deposition period, when all the seated particles behave elastically. The time domain is defined by the interval $t \in\left[0, T^{e}\right]$ and the spatial domain extends through $x_{3} \in[0$, $H(t)]$. This phase ends when the stress of the particles located at $x_{3}=0$ reaches $f^{p}\left(\underline{\underline{\sigma}}, p_{c 0}\right)=0$ at time $t=T^{e}$. At that moment, the height of the basin is $H\left(T^{e}\right)=H^{e}$.

- Elastic-plastic phase: In this phase, the upper part of the basin exhibits elastic behavior whereas the other part presents elastoplastic behavior. The time domain corresponds to the interval $t \in\left[T^{e}, T^{p}\right]$. The elastic domain develops along $x_{3} \in\left[H(t)-H^{e}, H(t)\right]$, whereas the elastoplastic domain develops for $x_{3} \in\left[0, H(t)-H^{e}\right]$. This phase ends when

the stress of the particles located at $x_{3}=0$ reaches $f^{v p}\left(\underline{\underline{\sigma}}, p_{v p}\right)=0$ at time $t=T^{p}$. At that moment, the basin exhibits thickness $H\left(T^{p}\right)=H^{p}$.

- Elastic-plastic-viscoplastic phase: Starting from the top of the basin and moving downward, the basin displays in this phase three distinct layers - an elastic layer followed by an elastoplastic layer while the particles in the bottom layer 


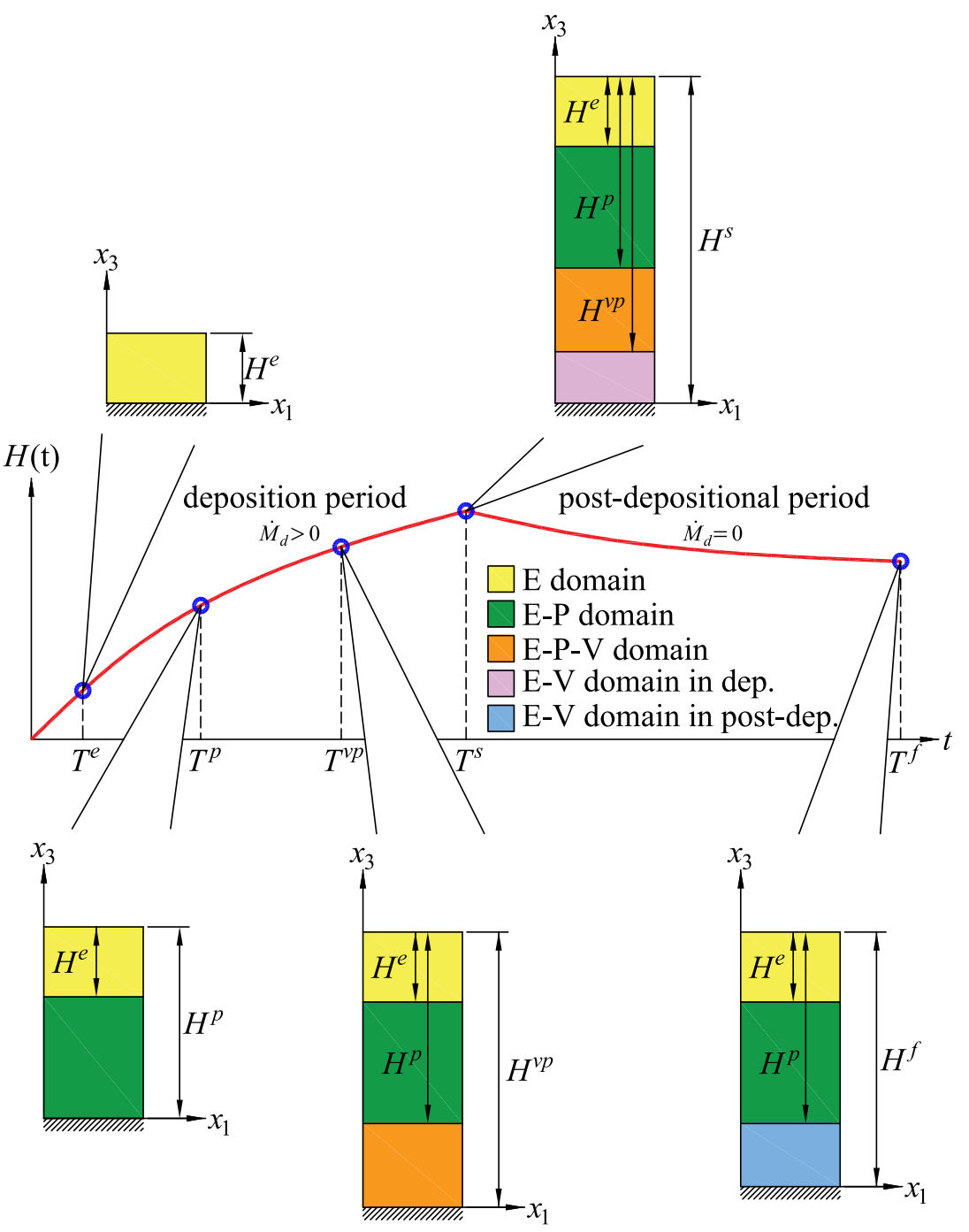

FI G U RE 4 Schematic representation of the evolution of the sedimentary basin during geological time [Colour figure can be viewed at wileyonlinelibrary.com]

undergo elastic-plastic-viscoplastic strains. This phase corresponds to time interval $t \in\left[T^{p}, T^{v p}\right]$. The elastic layer extends in the same spatial domain of the preceding phase $x_{3} \in\left[H(t)-H^{e}, H(t)\right]$. The elastoplastic layer is defined by $x_{3} \in\left[H(t)-H^{p}, H(t)-H^{e}\right]$ whereas the elastoplastic-viscoplastic one develops for $x_{3} \in\left[0, H(t)-H^{p}\right]$. This phase ends when the plastic part of strain of the particles located at $x_{3}=0$ no longer evolves $\left(\underline{\underline{d}}^{p}=\underline{\underline{0}}\right.$ or $\left.\dot{\chi}=0\right)$ at time $t=T^{v p}$. At that moment, the basin thickness is $H\left(T^{v p}\right)=H^{v p}$.

- Elastic-viscoplastic phase in sediment deposition period: In addition to three layers appearing in the previous phase, particles located at the bottom of the basin undergo purely elasto-viscoplastic strains. This phase takes place as long as be sediment accretion proceeds $t \in\left[T^{v p}, T^{s}\right]$, where $T^{s}$ stands for the prescribed duration of sediment deposition process. The elastic and elastoplastic behaviors develop in the same spatial domains appearing in preceding phase $x_{3} \in\left[H(t)-H^{e}, H(t)\right]$ and $x_{3} \in\left[H(t)-H^{p}, H(t)-H^{e}\right]$. The elastoplastic-viscoplastic layer extends in $x_{3} \in\left[H(t)-H^{v p}, H(t)-H^{p}\right]$, whereas the purely elasto-viscoplastic layer develops in $x_{3} \in[0$, $H(t)-H^{v p}$. The end of this phase coincides with that of sediment deposition period at time $t=T^{s}$. At that moment, the basin thickness is $H\left(T^{s}\right)=H^{s}$.

- Elastic-viscoplastic phase during postdepositional period: This phase starts at the beginning of the basin postdepositional period. The time domain corresponds to $t \geq T^{s}$. The elastic and elastic-plastic layers do not evolve and present the same thicknesses in the preceding phase. The elastic-viscoplastic behavior develops in domain $x_{3} \in[0$, $\left.H(t)-H^{p}\right]$. The end of this phase is defined by arbitrarily final time of analysis $T^{f}$ in which the basin reaches height $H\left(T^{f}\right)=H^{f}$. 
It is interesting to note that the layer thicknesses $H^{e}$ and $H^{p}$ remain constant along all the basin evolution for $t>T^{e}$. This means that after their formation, these layers translate upward parallel to direction $\underline{e}_{3}$ as the sediments are deposited.

The same observation holds regarding the layer thickness $H^{v p}$, which remains constant as long as the sediment accretion proceeds (i.e., for $T^{v p} \leq t \leq T^{s}$ ) and vanishes when it stops.

The system of differential equations that describe the sedimentary basin mechanical state is formed by the momentum balance 1 , the mass balance equation 3 , the relationship between velocity gradient and transformation gradient 18 , the relationship between the irreversible strain rate and the Jacobian irreversible component of the transformation 26 , and the constitutive behavior law 23. This system relates the unknown fields $\Lambda, J_{i r}, \sigma_{h}, \sigma_{v}$, and $u_{3}$ of the problem. It corresponds to a first-order nonlinear partial differential system of equations. These equations are completed by the complementary constitutive relationships as well as by initial and boundary conditions.

In Figure 5, two snapshots are presented in order to illustrate the sedimentary basin formulation. The first one corresponds to elastic-viscoplastic phase during the sediment deposition period. The second one refers to the nondepositional period. Strain rates relevant to the formulation in each phase are also presented.

\section{1 | Problem resolution for the accretion period $\dot{M}_{d}>0$}

During the depositional period, the material domain is evolving due to continuous sediment supply at the top of the basin. The sedimentary basin can thus be viewed as an open material system. An Eulerian description of particle motion is therefore suitable.

Referring to the mechanical state of the basin, closed-form solutions prove difficult to derive. Only semianalytical will be elaborated for the system of differential equations controlling the field variables in the sedimentarybasin.

Prior to further developments, it is assumed that the sedimentation process remains monotonic along the whole depositional phase (i.e., $\dot{M}_{d}>0$ ), thus excluding in particular any erosion period. Under this assumption, the stress and strain fields can conveniently be regarded as a function of the vertical stretch. In particular, the stress can be written as

$$
\underline{\underline{\sigma}}\left(x_{3}, t\right)=\underline{\underline{\sigma}}\left(\Lambda\left(x_{3}, t\right)\right)
$$

Such an observation allows for a simplified formulation of the PDE governing the mechanical evolution of the basin. From a computational viewpoint, the resolution procedure is performed incrementally through time discretization of governing equations. Starting from basin configuration known at time $t$, the problem amounts to numerically solving for each time step $\Delta t$ an approximate ODE system stated on the material system with known thickness $H(t)$. The procedure allows thus for the determination of the new basin configuration at time $t+\Delta t$. In particular, the thickness basin is updated using Equation 13 in discretized form:

FI G URE 5 Illustrative representation of sedimentary basin layers behavior [Colour figure can be viewed at wileyonlinelibrary.com]
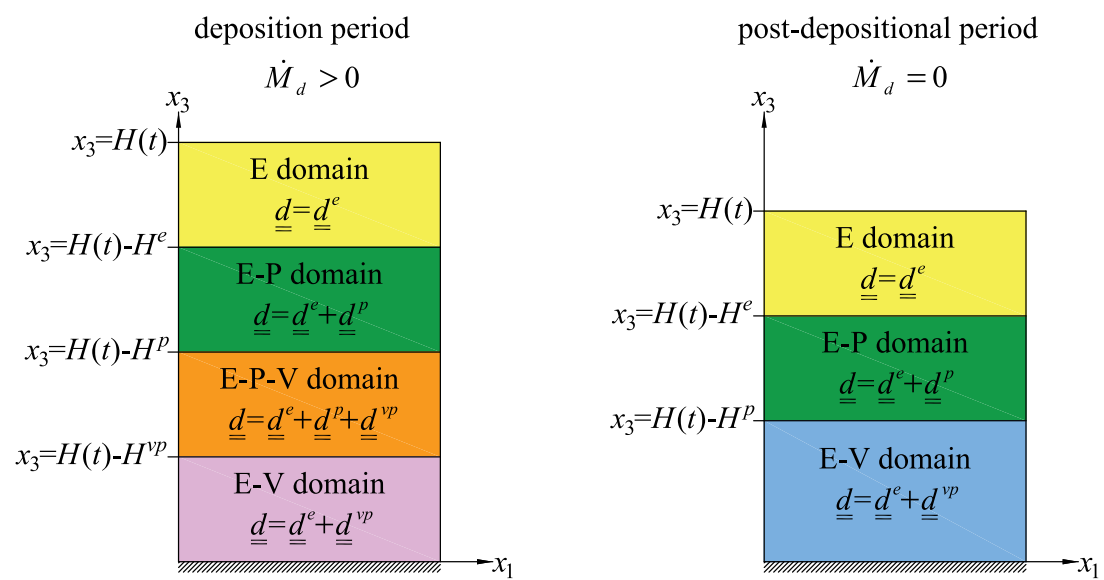


$$
H(t+\Delta t)=H(t)+\Delta H \text { with } \Delta H \simeq\left(\dot{M}_{d}(t) / \rho_{0}+u_{3}(H(t), t)\right) \Delta t
$$

In the time incremental procedure adopted for resolution, continuity conditions for all relevant fields should be accounted for at the interfaces of layers $x_{3}=H(t)-H^{e}, x_{3}=H(t)-H^{p}$, and $x_{3}=H(t)-H^{v p}$.

The incremental scheme as well as the solution to the system of ordinary nonlinear differential equations is performed numerically using the MAPLE software. A finite difference technique with Richardson extrapolation is used to solve the BVP.

\subsection{1 | Elastic phase}

At the beginning of the process, the thickness of the basin is $H(0)=0$. It then progressively increases as sediments are brought. The strain induced by gravity effects remains elastic until the thickness reaches a threshold $H^{e}$ defined below. The absence of irreversible strain $\left(J_{i r}=1\right.$ and $\left.\dot{J}_{i r}=0\right)$ implies that elastic moduli remain constant, thus simplifying the constitutive law 23.

For the elastic domain $\left(\underline{\underline{d}}^{i r}=\underline{\underline{0}}\right)$, the simplified constitutive law provides

$$
\begin{aligned}
& \dot{\sigma}_{h}=F_{h}^{e} \frac{\dot{\Lambda}}{\Lambda}, \\
& \dot{\sigma}_{v}=F_{v}^{e} \frac{\dot{\Lambda}}{\Lambda},
\end{aligned}
$$

where $F_{h}^{e}=K-2 / 3 \mu$ and $F_{v}^{e}=K+4 / 3 \mu$. These equations have 4 and 5 as the initial conditions.

Integrating Expressions 37 and 38 yields

$$
\begin{gathered}
\sigma_{h}=F_{h}^{e} \ln \Lambda, \\
\sigma_{v}=F_{v}^{e} \ln \Lambda .
\end{gathered}
$$

It follows from the momentum balance equation 1 that:

$$
\frac{\partial \sigma_{v}}{\partial x_{3}}=\frac{\rho_{0} g}{\Lambda}
$$

Substituting 40 into the momentum balance equation 41 leads to

$$
\frac{\partial \Lambda}{\partial x_{3}}=\frac{\rho_{0} g}{F_{v}^{e}} .
$$

Integrating the previous equation with the account for the initial condition 14 provides

$$
\Lambda\left(x_{3}, t\right)=1-\frac{\rho_{0} g}{F_{v}^{e}}\left(H(t)-x_{3}\right) .
$$

Combining Equations 20 and 38, we can rewrite the relation 17 as

$$
\frac{\partial u_{3}}{\partial x_{3}}=\frac{-\dot{M}_{d} g}{F_{v}^{e}}
$$


whose integrating with the boundary condition 7 yields

$$
u_{3}(H(t), t)=\frac{-\dot{M}_{d} g}{F_{v}^{e}} H(t)
$$

By substituting the previous equation into Equation 13 and then integrating it with the initial condition $H(0)=0$, we obtain the gravitational compaction law in the elastic phase:

$$
H(t)=\frac{F_{v}^{e}}{\rho_{0} g}\left(1-\exp \left(-\frac{g}{F_{v}^{e}} M_{d}(t)\right)\right) .
$$

This phase ends when the stress of the particles located at $x_{3}=0$ reaches $f^{p}\left(\underline{\underline{\sigma}}, p_{c 0}\right)=0$ at time $t=T^{e}$. At this moment, the basin is characterized by

$$
\left\{\begin{array}{l}
\Lambda^{e}=\Lambda\left(0, T^{e}\right)=e^{-\frac{p_{c 0}}{K+\frac{2 \sqrt{3}}{3} \mu a}} \\
H^{e}=H\left(T^{e}\right)=\frac{F_{v}^{e}}{\rho_{0} g}\left(1-\Lambda^{e}\right) \\
\sigma_{h}^{e}=\sigma_{h}\left(0, T^{e}\right)=-\frac{F_{h}^{e} p_{c 0}}{K+\frac{2 \sqrt{3}}{3} \mu a} \\
\sigma_{v}^{e}=\sigma_{v}\left(0, T^{e}\right)=-\frac{F_{v}^{e} p_{c 0}}{K+\frac{2 \sqrt{3}}{3} \mu a} \\
u_{3}^{e}=u_{3}\left(H^{e}, T^{e}\right)=\frac{-\dot{M}_{d} g}{F_{v}^{e}} H^{e}
\end{array}\right.
$$

where the time $T^{e}$ is obtained from relationship $H^{e}=H\left(T^{e}\right)$, which can equivalently be expressed through

$$
M_{d}\left(T^{e}\right)=\frac{F_{v}^{e}}{g} \frac{p_{c 0}}{K+\frac{2 \sqrt{3}}{3} \mu a} .
$$

\subsection{2 | Elastic-plastic phase}

As burial proceeds for $t \geq T^{e}$, elastoplastic strains develop in the deeper layers of the basin, that is, when the thickness of the basin becomes greater than the threshold $H^{e}$. The thickness of the elastic layer remains constant in time, equal to $H^{e}$, and extends in the range $x_{3} \in\left[H(t)-H^{e}, H(t)\right]$ (Figure 4). In contrast, the thickness of the elastoplastic layer increases from bottom $x_{3}=0$ as the sediments are supplied. The domain is defined at each instant by the range $x_{3} \in[0$, $\left.H(t)-H^{e}\right]$.

The plastic strain rate 28 is rewritten considering the plastic yield condition 27:

$$
\underline{\underline{d}}^{p}=\dot{\chi} \frac{\partial f^{p}}{\partial \underline{\underline{\sigma}}}=\dot{\chi}\left(-\frac{1}{3} \underline{\underline{1}}+\frac{a}{2 \sqrt{J_{2}}} \underline{\underline{S}}\right) .
$$

Relationship 26 applied to the previous equation results in 


$$
\operatorname{tr}_{\underline{\underline{d}}}^{i r}=\frac{\dot{J}_{i r}}{J_{i r}}=-\dot{\chi}
$$

Introducing the strain rate 18 , the material elastic stiffness moduli tensor 24 , and the plastic strain rate 49 into the constitutive behavior law 23 yields

$$
\begin{aligned}
& \underline{\underline{\dot{\sigma}}}=\left[\beta_{h}^{e} \frac{\dot{\Lambda}}{\Lambda}+\left(K-\frac{\sqrt{3}}{3} \mu a\right) \dot{\chi}+\frac{\operatorname{tr} \underline{\underline{\sigma}}}{3} \frac{\dot{K}}{K}-\frac{\left(\sigma_{v}-\sigma_{h}\right)}{3} \frac{\dot{\mu}}{\mu}\right]\left(\underline{e}_{1} \otimes \underline{e}_{1}+\underline{e}_{2} \otimes \underline{e}_{2}\right) \\
& +\left[\beta_{v}^{e} \frac{\dot{\Lambda}}{\Lambda}+\left(K+\frac{2 \sqrt{3}}{3} \mu a\right) \dot{\chi}+\frac{\operatorname{tr} \underline{\underline{\sigma}}}{3} \frac{\dot{K}}{K}+\frac{2\left(\sigma_{v}-\sigma_{h}\right)}{3} \frac{\dot{\mu}}{\mu}\right] \underline{e}_{3} \otimes \underline{e}_{3} .
\end{aligned}
$$

The consistency condition $\dot{f}^{p}=0$ expressed for the yield criterion 27 gives

$$
\dot{f}^{p}=\frac{\partial f^{p}}{\partial \underline{\underline{\sigma}}}: \underline{\underline{\dot{\sigma}}}-\dot{p}_{c}=-\frac{1}{3} \operatorname{tr} \underline{\underline{\dot{\sigma}}}+\frac{a}{2 \sqrt{J_{2}}} \underline{\underline{S}}: \underline{\underline{\dot{S}}}-\dot{p}_{c}=0
$$

Based on the above equation, it is possible to determine the expression of the plastic multiplier:

$$
\dot{\chi}=G_{1}^{p}\left(J_{i r}\right) \frac{\dot{\Lambda}}{\Lambda}+G_{2}^{p}\left(J_{i r}\right) \dot{J}_{i r} \text { with }\left\{\begin{array}{l}
G_{1}^{p}\left(J_{i r}\right)=-\frac{K+\frac{2 \sqrt{3}}{3} \mu a}{K+a^{2} \mu+h_{p}} \\
G_{2}^{p}\left(J_{i r}\right)=-\frac{\left(\sigma_{v}+2 \sigma_{h}\right) \bar{K}+a \sqrt{3}\left(\sigma_{v}-\sigma_{h}\right) \bar{\mu}}{K+a^{2} \mu+h_{p}}
\end{array}\right.
$$

where the nondimensional stiffness parameters $\bar{K}$ and $\bar{\mu}$ are defined by $\bar{K}=\frac{1}{3 K} \frac{\partial K}{\partial J_{i r}}$ and $\bar{\mu}=\frac{1}{3 \mu} \frac{\partial \mu}{\partial J_{i r}}$. Note that $h_{p}=h_{p}(\varphi)=h_{p}\left(J_{i r}\right)$ by virtue of 30 .

It follows from 21 and 22 that

$$
\left\{\begin{array}{l}
\bar{K}=-\frac{1}{3} \frac{3 k^{s}+4 \mu^{s}}{J_{i r}\left(3 k^{s}+4 \mu^{s}\right)-3 k^{s}\left(1-\phi_{0}\right)} \\
\bar{\mu}=-\frac{5}{3} \frac{3 k^{s}+4 \mu^{s}}{3 k^{s}\left(5 J_{i r}-2\left(1-\phi_{0}\right)\right)+4 \mu^{s}\left(5 J_{i r}-3\left(1-\phi_{0}\right)\right)}
\end{array}\right.
$$

Substituting Equation 53 into 51 yields

$$
\underline{\underline{\sigma}}=\left[F_{h 1}^{p} \frac{\dot{\Lambda}}{\Lambda}+F_{h 2}^{p} \dot{J}_{i r}\right]\left(\underline{e}_{1} \otimes \underline{e}_{1}+\underline{e}_{2} \otimes \underline{e}_{2}\right)+\left[F_{v 1}^{p} \frac{\dot{\Lambda}}{\Lambda}+F_{v 2}^{p} \dot{J}_{i r}\right] \underline{e}_{3} \otimes \underline{e}_{3}
$$

where

$$
\left\{\begin{array}{l}
F_{h 1}^{p}\left(J_{i r}\right)=\beta_{h}^{e}+\left(K-\frac{\sqrt{3}}{3} \mu a\right) G_{1}^{p} \\
F_{h 2}^{p}\left(J_{i r}\right)=\left(K-\frac{\sqrt{3}}{3} \mu a\right) G_{2}^{p}+\left(\sigma_{v}+2 \sigma_{h}\right) \bar{K}-\left(\sigma_{v}-\sigma_{h}\right) \bar{\mu} \\
F_{v 1}^{p}\left(J_{i r}\right)=\beta_{v}^{e}+\left(K+\frac{2 \sqrt{3}}{3} \mu a\right) G_{1}^{p} \\
F_{v 2}^{p}\left(J_{i r}\right)=\left(K+\frac{2 \sqrt{3}}{3} \mu a\right) G_{2}^{p}+\left(\sigma_{v}+2 \sigma_{h}\right) \bar{K}+2\left(\sigma_{v}-\sigma_{h}\right) \bar{\mu}
\end{array} .\right.
$$


Based on observation 35, Equation 55 can be rewritten as

$$
\frac{\partial \underline{\underline{\sigma}}}{\partial x_{3}}=\left[\frac{F_{h 1}^{p}}{\Lambda} \frac{\partial \Lambda}{\partial x_{3}}+F_{h 2}^{p} \frac{\partial J_{i r}}{\partial x_{3}}\right]\left(\underline{e}_{1} \otimes \underline{e}_{1}+\underline{e}_{2} \otimes \underline{e}_{2}\right)+\left[\frac{F_{v 1}^{p}}{\Lambda} \frac{\partial \Lambda}{\partial x_{3}}+F_{v 2}^{p} \frac{\partial J_{i r}}{\partial x_{3}}\right] \underline{e}_{3} \otimes \underline{e}_{3} .
$$

Combining the momentum balance equation 41 and the vertical component of stress in 57 leads to

$$
\frac{\partial \Lambda}{\partial x_{3}}=\frac{\rho_{0} g}{F_{v 1}^{p}}-\frac{F_{v 2}^{p}}{F_{v 1}^{p}} \Lambda \frac{\partial J_{i r}}{\partial x_{3}}
$$

Recalling that $J_{i r}\left(x_{3}, t\right)=J_{i r}\left(\Lambda\left(x_{3}, t\right)\right)$, it follows from 50 and 53 that

$$
\frac{\partial J_{i r}}{\partial x_{3}}=-\frac{G_{1}^{p} J_{i r}}{\Lambda\left(1+G_{2}^{p} J_{i r}\right)} \frac{\partial \Lambda}{\partial x_{3}} .
$$

Combining Equations 17 and 20 with the expression of vertical stress rate given by 55 yields the law of spatial variation of particles velocity

$$
\frac{\partial u_{3}}{\partial x_{3}}=\frac{-\dot{M}_{d} g}{F_{v 1}^{p}+F_{v 2}^{p} \Lambda \frac{\partial J_{i r}}{\partial x_{3}} / \frac{\partial \Lambda}{\partial x_{3}}},
$$

which should be completed with the boundary condition 7 .

Equations 57-59 governing the stress and strain fields, together with Equation 60 related to particles velocity field, define the system of differential equations governing mechanical state of the elastoplastic layer of the sedimentary basin $0 \leq x_{3} \leq H(t)-H^{e}$. The continuity conditions at the interface with the upper elastic layer $H(t)-H^{e} \leq x_{3} \leq H(t)$ are expressed from the quantities computed at the end of elastic phase through 47: $\Lambda\left(H(t)-H^{e}, t\right)=\Lambda^{e}, \underline{\sigma}\left(H(t)-H^{e}, t\right)=\underline{\sigma}^{e}$, and $J_{i r}\left(H(t)-H^{e}, t\right)=1$. Such a mathematical problem is not tractable analytically and is solved numerically for each time step using MAPLE software. The end of this phase corresponds to time $t=T^{p}$ at which the stress of material particles located at $x_{3}=0$ complies with viscoplasticity condition $f^{v p}\left(\underline{\underline{\sigma}}, p_{v p}\right)=0$. At that moment, the thickness of the basin is $H^{p}=H\left(T^{p}\right)$, and the following quantities are computed:

$$
\left\{\begin{array}{l}
\Lambda^{p}=\Lambda\left(0, T^{p}\right) \\
\sigma_{h}^{p}=\sigma_{h}\left(0, T^{p}\right) \\
\sigma_{v}^{p}=\sigma_{v}\left(0, T^{p}\right) \\
J_{i r}^{p}=J_{i r}\left(0, T^{p}\right) \\
u_{3}^{p}=u_{3}\left(H^{p}-H^{e}, T^{p}\right)
\end{array}\right.
$$

and will be used as "boundary conditions" at $x_{3}=H^{p}-H^{e}$ for the next elastic-plastic-viscoplastic layer that develops for $t \geq T^{p}$.

It is noted that the velocity of particles located at the top of the basin is given by

$$
u_{3}(H(t), t)=u_{3}^{e}+u_{3}\left(H(t)-H^{e}, t\right) .
$$

\subsection{3 | Elastic-plastic-viscoplastic phase}

The response of the basin in the elastic-plastic-viscoplastic phase should be analyzed for $t \geq T^{p}$ when the thickness of the basin becomes higher than the threshold $H^{p}$. As for the elastic domain, the thickness of the elastoplastic layer 
$x_{3} \in\left[H(t)-H^{p}, H(t)-H^{e}\right]$ (Figure 4) remains constant in time. In contrast, the thickness of the elastoplasticviscoplastic layer increases from bottom $x_{3}=0$ as the sediments are supplied. This material domain is defined by the range $x_{3} \in\left[0, H(t)-H^{p}\right]$.

Relationship 26 applied to the plastic strain rate 28 and the viscoplastic strain rate 32 results in

$$
\operatorname{tr}_{\underline{\underline{d}}}^{i r}=\frac{\dot{J}_{i r}}{J_{i r}}=-\left(\dot{\chi}+\frac{f^{v p}}{\eta}\right)=-\Psi .
$$

The constitutive equation 23 reads

$$
\begin{aligned}
& \underline{\underline{\sigma}}=\left[\beta_{h}^{e} \frac{\dot{\Lambda}}{\Lambda}+\left(K-\frac{\sqrt{3}}{3} \mu a\right) \Psi+\frac{\operatorname{tr} \underline{\underline{\sigma}}}{3} \frac{\dot{K}}{K}-\frac{\left(\sigma_{v}-\sigma_{h}\right)}{3} \frac{\dot{\mu}}{\mu}\right]\left(\underline{e}_{1} \otimes \underline{e}_{1}+\underline{e}_{2} \otimes \underline{e}_{2}\right) \\
& +\left[\beta_{v}^{e} \frac{\dot{\Lambda}}{\Lambda}+\left(K+\frac{2 \sqrt{3}}{3} \mu a\right) \Psi+\frac{\operatorname{tr} \underline{\underline{\sigma}}}{3} \frac{\dot{K}}{K}+\frac{2\left(\sigma_{v}-\sigma_{h}\right)}{3} \frac{\dot{\mu}}{\mu}\right] \underline{e}_{3} \otimes \underline{e}_{3} .
\end{aligned}
$$

It follows from the consistency condition $\dot{f}^{p}=0$ that

$$
\Psi=G_{1}^{v p}\left(J_{i r}\right) \frac{\dot{\Lambda}}{\Lambda}+G_{2}^{v p}\left(J_{i r}\right) \dot{J}_{i r} \quad \text { with }\left\{\begin{array}{l}
G_{1}^{v p}\left(J_{i r}\right)=-\frac{K+\frac{2 \sqrt{3}}{3} \mu a}{K+a^{2} \mu+h_{p}} \\
G_{2}^{v p}\left(J_{i r}\right)=-\frac{\left(\sigma_{v}+2 \sigma_{h}\right) \bar{K}+a \sqrt{3}\left(\sigma_{v}-\sigma_{h}\right) \bar{\mu}}{K+a^{2} \mu+h_{p}}
\end{array} .\right.
$$

The system that describes the mechanical behavior of the basin in the elastoplastic-viscoplastic domain at each instant is given by

$$
\left\{\begin{array}{l}
\frac{\partial \Lambda}{\partial x_{3}}=\frac{\rho_{0} g}{F_{v 1}^{v p}}-\frac{F_{v 2}^{v p}}{F_{v 1}^{v p}} \Lambda \frac{\partial J_{i r}}{\partial x_{3}} \\
\frac{\partial \sigma_{h}}{\partial x_{3}}=\frac{F_{h 1}^{v p}}{\Lambda} \frac{\partial \Lambda}{\partial x_{3}}+F_{h 2}^{v p} \frac{\partial J_{i r}}{\partial x_{3}} \\
\frac{\partial \sigma_{v}}{\partial x_{3}}=\frac{F_{v 1}^{v p} \frac{\partial \Lambda}{\Lambda}}{\partial x_{3}}+F_{v 2}^{v p} \frac{\partial J_{i r}}{\partial x_{3}} \\
\frac{\partial J_{i r}}{\partial x_{3}}=-\frac{G_{1}^{v p} J_{i r}}{\Lambda\left(1+G_{2}^{v p} J_{i r}\right.} \frac{\partial \Lambda}{\partial x_{3}} \\
\frac{\partial u_{3}}{\partial x_{3}}=\frac{-\dot{M}_{d} g}{F_{v 1}^{v p}+F_{v 2}^{v p} \Lambda \frac{\partial J_{i r}}{\partial x_{3}} / \frac{\partial \Lambda}{\partial x_{3}}}
\end{array}\right.
$$

where

$$
\left\{\begin{array}{l}
F_{h 1}^{v p}\left(J_{i r}\right)=\beta_{h}^{e}+\left(K-\frac{\sqrt{3}}{3} \mu a\right) G_{1}^{v p} \\
F_{h 2}^{v p}\left(J_{i r}\right)=\left(K-\frac{\sqrt{3}}{3} \mu a\right) G_{2}^{v p}+\left(\sigma_{v}+2 \sigma_{h}\right) \bar{K}-\left(\sigma_{v}-\sigma_{h}\right) \bar{\mu} \\
F_{v 1}^{v p}\left(J_{i r}\right)=\beta_{v}^{e}+\left(K+\frac{2 \sqrt{3}}{3} \mu a\right) G_{1}^{v p} \\
F_{v 2}^{v p}\left(J_{i r}\right)=\left(K+\frac{2 \sqrt{3}}{3} \mu a\right) G_{2}^{v p}+\left(\sigma_{v}+2 \sigma_{h}\right) \bar{K}+2\left(\sigma_{v}-\sigma_{h}\right) \bar{\mu}
\end{array} .\right.
$$


Equations 66 should be completed by $\Lambda\left(H(t)-H^{p}, t\right)=\Lambda^{p}, \underline{\underline{\sigma}}\left(H(t)-H^{p}, t\right)=\underline{\underline{\sigma}}^{p}$, and $J_{i r}\left(H(t)-H^{p}, t\right)=J_{i r}^{p}$ representing the conditions of continuity at the interface between the elastoplastic and elastoplasticviscoplastic domains.Finally, integration of $u_{3}$ in 66 requires to account for Condition 7 that prescribes the velocity at $x_{3}=0$.

The thickness of the elastoplastic-viscoplastic layer is increasing with time as long as $\underline{\underline{d}}^{p} \neq \underline{\underline{0}}$ and $\underline{\underline{d}}^{v p} \neq \underline{\underline{0}}$. This phase ends in time $t=T^{v p}$ when the plastic component $\underline{\underline{d}}^{p}$ of strain rate vanishes for particles located at $x_{3}=0$. This means that $\dot{\chi}=0$ and no additional plastic strains are therefore developed. At $t=T^{v p}$, the basin thickness is $H\left(T^{v p}\right)=H^{v p}$ and the following quantities are computed:

$$
\left\{\begin{array}{l}
\Lambda^{v p}=\Lambda\left(0, T^{v p}\right) \\
\sigma_{h}^{v p}=\sigma_{h}\left(0, T^{v p}\right) \\
\sigma_{v}^{v p}=\sigma_{v}\left(0, T^{v p}\right) \\
J_{i r}^{v p}=J_{i r}\left(0, T^{v p}\right) \\
u_{3}^{v p}=u_{3}\left(H^{p}-H^{e}, T^{v p}\right)
\end{array} .\right.
$$

Along this phase, the velocity at the top of the basin is given by

$$
u_{3}(H(t), t)=u_{3}^{e}+u_{3}^{p}+u_{3}\left(H(t)-H^{p}, t\right)
$$

\subsection{4 | Elastic-viscoplastic phase}

As accretion proceeds, a new layer referred to as elastic-viscoplastic layer starts developing for $t \geq T^{v p}$ in the material domain $0 \leq x_{3} \leq H(t)-H^{v p}$ (Figure 4). In the latter, the plastic strain in not evolving so that $\underline{\underline{d}}^{p}=0$, and, therefore, $\underline{\underline{d}}^{i r}=\underline{\underline{d}}^{v p}$. Elastic, plastic, and plastic-viscoplastic layers keep constant thicknesses along this phase, equal, respectively, to $H^{e}, H^{p}-H^{e}$, and $H^{v p}-H^{p}$.

On the one hand, state equation 23 together with the viscoplastic flow rule 32 provides

$$
\begin{aligned}
& \underline{\underline{\sigma}}=\left[\beta_{h}^{e} \frac{\dot{\Lambda}}{\Lambda}+\left(K-\frac{\sqrt{3}}{3} \mu a\right) \frac{f^{v p}}{\eta}+\frac{\operatorname{tr} \underline{\underline{\sigma}}}{3} \frac{\dot{K}}{K}-\frac{\left(\sigma_{v}-\sigma_{h}\right)}{3} \frac{\dot{\mu}}{\mu}\right]\left(\underline{e}_{1} \otimes \underline{e}_{1}+\underline{e}_{2} \otimes \underline{e}_{2}\right) \\
& +\left[\beta_{v}^{e} \frac{\dot{\Lambda}}{\Lambda}+\left(K+\frac{2 \sqrt{3}}{3} \mu a\right) \frac{f^{v p}}{\eta}+\frac{\operatorname{tr} \underline{\underline{\sigma}} \dot{K}}{3} \frac{2\left(\sigma_{v}-\sigma_{h}\right)}{3} \frac{\dot{\mu}}{\mu}\right] \underline{e}_{3} \otimes \underline{e}_{3} .
\end{aligned}
$$

On the other hand, Expression 26 of irreversible volume dilatation expresses in the elastic-viscoplastic layer as

$$
\operatorname{tr} \underline{\underline{d}}^{i r}=\frac{\dot{J}_{i r}}{J_{i r}}=-\frac{f^{v p}}{\eta}
$$

Such a differential equation linking $J_{i r}$ and $\Lambda$ to stress state $\underline{\underline{\sigma}}$ proves difficult to be handled numerically in the context of Maple procedures. For this reason and in order to preserve semianalytical formulation of the problem solution, an uncontrolled approximation shall be introduced at this stage. It consists in assuming along each time step that $\dot{f}^{v p}=0$. In the time incremental procedure adopted for solving the differential equations governing the mechanical fields in the elastic-viscoplastic layer, this approximation amounts to considering that the value of $f^{p}$ attached to each particle is piecewise constant with respect to time. Such an approximation yields 


$$
-\frac{\dot{J}_{i r}}{J_{i r}}=G_{1}^{v}\left(J_{i r}\right) \frac{\dot{\Lambda}}{\Lambda}+G_{2}^{v}\left(J_{i r}\right) \dot{J}_{i r} \text { with }\left\{\begin{array}{l}
G_{1}^{v}\left(J_{i r}\right)=-\frac{K+\frac{2 \sqrt{3}}{3} \mu a}{K+a^{2} \mu+h_{v p}} \\
G_{2}^{v}\left(J_{i r}\right)=-\frac{\left(\sigma_{v}+2 \sigma_{h}\right) \bar{K}+a \sqrt{3}\left(\sigma_{v}-\sigma_{h}\right) \bar{\mu}}{K+a^{2} \mu+h_{v p}}
\end{array},\right.
$$

in which $h_{v p}=h_{v p}(\varphi)=h_{v p}\left(J_{i r}\right)$ as indicated in 34 .

The relevancy of such approximation as well as associated accuracy will be assessed a posteriori in Section 4, through comparison of the predicted solutions with those derived from a FE tool specifically devised for sedimentary basin simulation.

Substituting Expressions 71 and 72 into 70 yields

$$
\underline{\underline{\sigma}}=\left[F_{h 1}^{v} \frac{\dot{\Lambda}}{\Lambda}+F_{h 2}^{v} \dot{J}_{i r}\right]\left(\underline{e}_{1} \otimes \underline{e}_{1}+\underline{e}_{2} \otimes \underline{e}_{2}\right)+\left[F_{v 1}^{v} \frac{\dot{\Lambda}}{\Lambda}+F_{v 2}^{v} \dot{J}_{i r}\right] \underline{e}_{3} \otimes \underline{e}_{3}
$$

where

$$
\left\{\begin{array}{l}
F_{h 1}^{v}\left(J_{i r}\right)=\beta_{h}^{e}+\left(K-\frac{\sqrt{3}}{3} \mu a\right) G_{1}^{v} \\
F_{h 2}^{v}\left(J_{i r}\right)=\left(K-\frac{\sqrt{3}}{3} \mu a\right) G_{2}^{v}+\left(\sigma_{v}+2 \sigma_{h}\right) \bar{K}-\left(\sigma_{v}-\sigma_{h}\right) \bar{\mu} \\
F_{v 1}^{v}\left(J_{i r}\right)=\beta_{v}^{e}+\left(K+\frac{2 \sqrt{3}}{3} \mu a\right) G_{1}^{v} \\
F_{v 2}^{v}\left(J_{i r}\right)=\left(K+\frac{2 \sqrt{3}}{3} \mu a\right) G_{2}^{v}+\left(\sigma_{v}+2 \sigma_{h}\right) \bar{K}+2\left(\sigma_{v}-\sigma_{h}\right) \bar{\mu}
\end{array} .\right.
$$

A reasoning similar to that developed for the preceding phases of the basin evolution allows for the formulation of the ODE system that governs the mechanical state of the elastic-viscoplastic layer. It follows

$$
\left\{\begin{array}{l}
\frac{\partial \Lambda}{\partial x_{3}}=\frac{\rho_{0} g}{F_{v 1}^{v}}-\frac{F_{v 2}^{v}}{F_{v 1}^{v}} \Lambda \frac{\partial J_{i r}}{\partial x_{3}} \\
\frac{\partial \sigma_{h}}{\partial x_{3}}=\frac{F_{h 1}^{v}}{\Lambda} \frac{\partial \Lambda}{\partial x_{3}}+F_{h 2}^{v} \frac{\partial J_{i r}}{\partial x_{3}} \\
\frac{\partial \sigma_{v}}{\partial x_{3}}=\frac{F_{v 1}^{v}}{\Lambda} \frac{\partial \Lambda}{\partial x_{3}}+F_{v 2}^{v} \frac{\partial J_{i r}}{\partial x_{3}} \\
\frac{\partial J_{i r}}{\partial x_{3}}=-\frac{G_{1}^{v} J_{i r}}{\Lambda\left(1+G_{2}^{v} J_{i r}\right)} \frac{\partial \Lambda}{\partial x_{3}} \\
\frac{\partial u_{3}}{\partial x_{3}}=\frac{-\dot{M}_{d} g}{F_{v 1}^{v}+F_{v 2}^{v} \Lambda \frac{\partial J_{i r}}{\partial x_{3}} / \frac{\partial \Lambda}{\partial x_{3}}}
\end{array} .\right.
$$

The above equations should be completed by the continuity conditions $\Lambda\left(H(t)-H^{v p}, t\right)=\Lambda^{v p}, \underline{\underline{\sigma}}\left(H(t)-H^{v p}, t\right)=\underline{\underline{\sigma}}^{v p}$, and $J_{i r}\left(H(t)-H^{v p}, t\right)=J_{i r}^{v p}$ expressed at the interface between elastic-plastic-viscoplastic layer and elastic-viscoplastic layer, together with the boundary equation 7 expressing nullity of velocity at the rigid substratum.

This phase takes place as long as sediment accretion proceeds. When the latter stops at time $t=T^{s}$, the sedimentary basin will evolve subsequently as a closed material system without additional sediment supply (nondepositional period). The numerical incremental procedure used to solve this problem based on MAPLE software allows to compute at time $t=T^{s}$ the total thickness of the basin $H^{s}=H\left(T^{s}\right)$ as well as the following quantities: 


$$
\left\{\begin{array}{l}
\Lambda^{s}=\Lambda\left(0, T^{s}\right) \\
\sigma_{h}^{s}=\sigma_{h}\left(0, T^{s}\right) \\
\sigma_{v}^{s}=\sigma_{v}\left(0, T^{s}\right) \\
J_{i r}^{s}=J_{i r}\left(0, T^{s}\right) \\
u_{3}^{s}=u_{3}\left(H^{s}-H^{v p}, T^{s}\right)
\end{array} .\right.
$$

Note that during this phase, the velocity of particles located at the top of the basin is given by

$$
u_{3}(H(t), t)=u_{3}^{e}+u_{3}^{p}+u_{3}^{v p}+u_{3}\left(H(t)-H^{v p}, t\right) .
$$

\section{2 | Preliminary illustrative numerical results}

Semianalytical solutions for the evolution of stress and strains during the accretion period are presented for the data provided in Section 4.1. The purpose herein is merely illustrative, a more comprehensive analysis being the object of Section 4.2 the respective analysis of the mechanical variables.

The compaction law $t \rightarrow H(t)$ is presented in Figure 6, where the consecutive phases related to behavior of the sedimentary layers are reported. The basin thickness at the end of accretion period $t=T^{s}=60$ My (millions of years) is about $H^{s}=2864.21 \mathrm{~m}$, which corresponds to as overall compaction ratio of $1-H^{s} / \mathcal{H} \cong 52.2 \%$ where $\mathcal{H}=6000 \mathrm{~m}$ is the total sediment height supplied during this period.

The times of activation of each phase as well as associated layers extents can also be observed from this figure:

$$
\left\{\begin{array}{l}
T^{e} \leq T^{p} \leq T^{v p} \leq T^{s} \\
H^{e} \leq H^{p} \leq H^{v p} \leq H^{s}
\end{array}\right.
$$

The profiles along basin depth of total Jacobian $J=\Lambda$ as well as its irreversible component $J_{i r}$ are plotted in Figure 7A for $t=T^{s}$. As expected, values of $J$ and $J_{i r}$ prove very close to each other, thus validating the assumption of infinitesimal elastic strains. It is also observed that $J \approx J_{i r} \approx 0.3$ along the basin bottom $x_{3}=0$, approaching the asymptotic value $J_{i r}^{c r}=1-\phi_{0}=0.28$.

The porosity profile along the basin thickness calculated at time $t=T^{s}$ is shown in Figure 7B. Reflecting the significant material densification associated with burial process, this important parameter decreases from $\varphi=\phi_{0}=0.72$ at the top of the basin to $\varphi \approx 0.08$ at the bottom $x_{3}=0$, which indicates the trend towards total pore closure. This behavior is consistent with the increasing intensity of the stress components with depth as observed in the profile of Figure 8.

\subsection{Problem resolution for the postaccretion period $\dot{M}_{d}=0$}

In the nondepositional period that takes place for $t>T^{s}$, the sedimentary basin behaves as a closed material system because no additional material is supplied at the top of basin. A Lagrangian description with respect to the configuration reached by the basin at the end of accretion process $t=T^{s}$ appears therefore convenient for addressing the basin deformation during this phase. In this framework, a sediment particle is referred to by means of its initial coordinate $X_{3}=x_{3}\left(T^{s}\right)$. In the current configuration of the basin, the particle position at time $t$ is then defined by $x_{3}\left(X_{3}\right.$, $t)=X_{3}+\xi_{3}\left(X_{3}, t\right)$, where $\underline{\xi}=\xi_{3}\left(X_{3}, t\right) \underline{e}_{3}$ is the displacement vector.

A main characteristic of basin deformation during the nondepositional period lies in the fact that strains do not evolve in the upper elastic and plastic layers. The strain field is actually evolving $(\underline{d} \neq 0)$ only in the layer involving viscoplastic deformation, that is, for $0 \leq X_{3} \leq H^{s}-H^{p}$. The latter layer whose thickness is decreasing in time owing to viscoplastic compaction will be referred to as elastic-viscoplastic layer.

The response of the elastic-viscoplastic layer is studied along nondepositional period $T^{s} \leq t \leq T^{f}$, where $T^{f}$ is the total geological time chosen for analysis.

It follows from $\dot{M}_{d}=0$ and relationship 20 that the vertical stress remains constant 


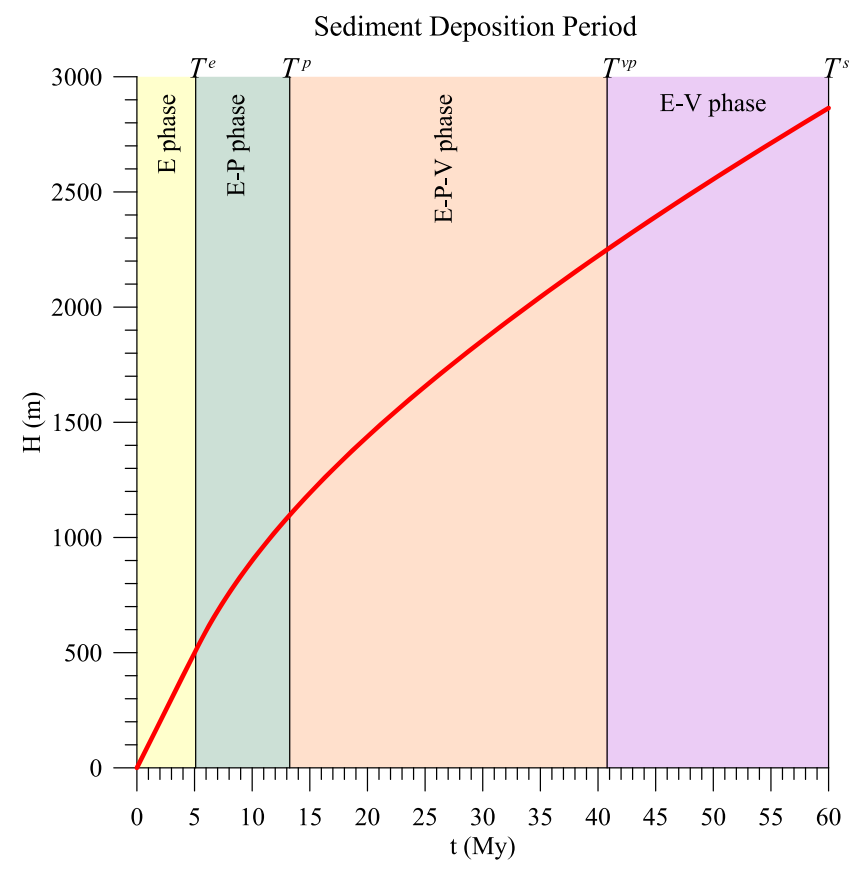

F I G U RE 6 Basin compaction law at the end of accretion period $t=T^{s}=60 \mathrm{My}$ [Colour figure can be viewed at wileyonlinelibrary.com]

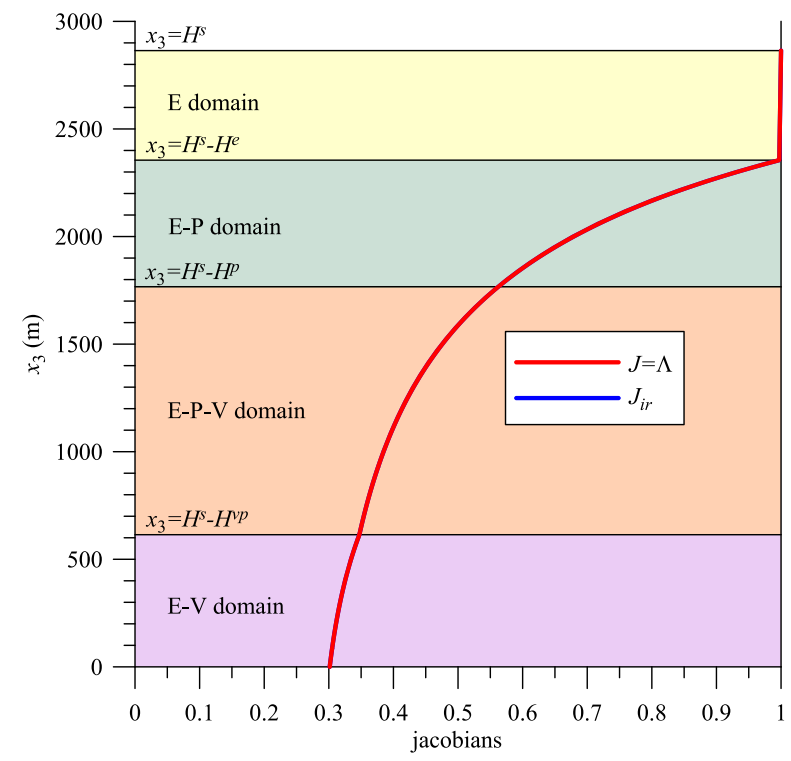

(A)

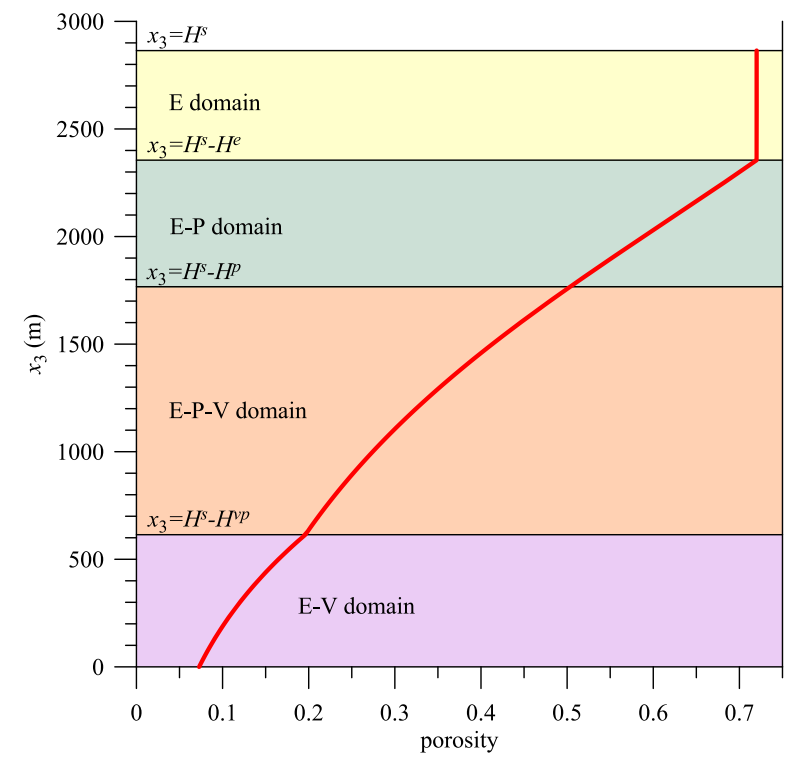

(B)

F I G URE 7 Profiles of the basin at $t=T^{s}=60 \mathrm{My}$ (end of accretion period): A, Jacobians of the transformation; B, Eulerian porosity [Colour figure can be viewed at wileyonlinelibrary.com]

$$
\dot{\sigma}_{v}=0
$$

whose integration between time $T^{s}$ and current time $t$ provides

$$
\sigma_{v}\left(X_{3}, t\right)=\sigma_{v}^{p s}\left(X_{3}\right),
$$

where $\sigma_{v}^{p s}\left(X_{3}\right)=\sigma_{v}\left(x_{3}=X_{3}, \mathrm{t}=T^{s}\right)$ is the known (calculated) vertical stress distribution that prevails in the basin at the end of accretion period $t=T^{s}$.

The constitutive state equation 23 in which $\underline{\underline{d}}^{i r}=\underline{\underline{d}}^{v p}$ is given by 32 yields 
F I G URE 8 Stress profiles of the basin at $t=T^{s}=60 \mathrm{My}$ (end of accretion period) [Colour figure can be viewed at wileyonlinelibrary. com]

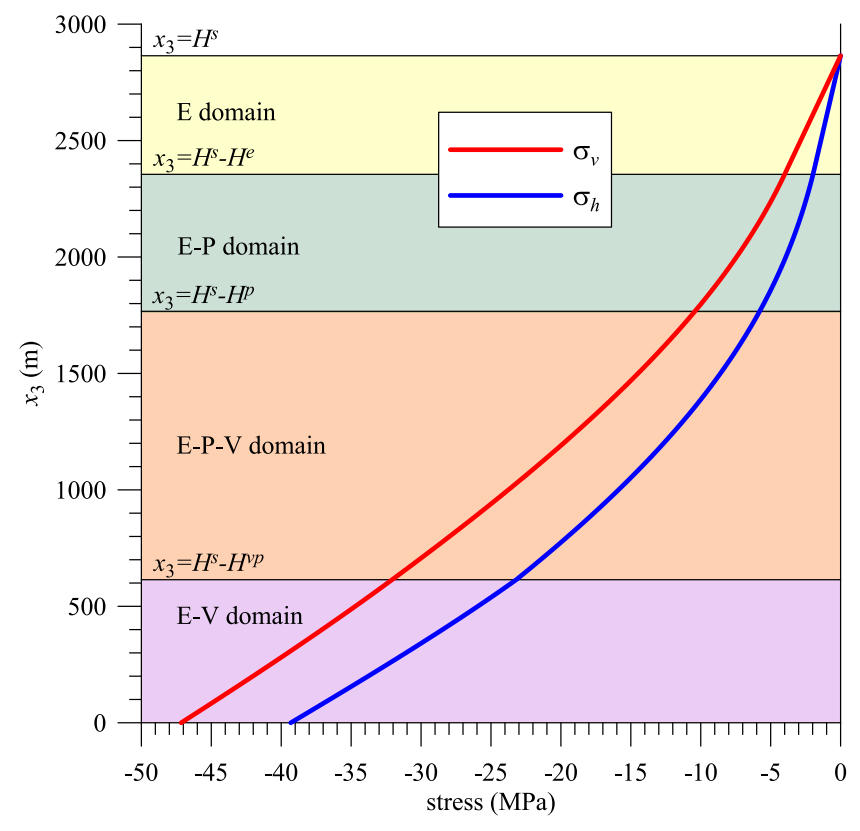

$$
\begin{aligned}
& \underline{\underline{\sigma}}=\left[F_{h}^{e} \frac{\dot{\Lambda}}{\Lambda}+\left(K-\frac{\sqrt{3}}{3} \mu a\right) \frac{f^{v p}}{\eta}+\frac{\operatorname{tr} \underline{\underline{\sigma}}}{3} \frac{\dot{K}}{K}-\frac{\left(\sigma_{v}-\sigma_{h}\right)}{3} \frac{\dot{\mu}}{\mu}\right]\left(\underline{e}_{1} \otimes \underline{e}_{1}+\underline{e}_{2} \otimes \underline{e}_{2}\right) \\
& +\left[F_{v}^{e} \frac{\dot{\Lambda}}{\Lambda}+\left(K+\frac{2 \sqrt{3}}{3} \mu a\right) \frac{f^{v p}}{\eta}+\frac{\operatorname{tr} \underline{\underline{\sigma}}}{3} \frac{\dot{K}}{K}+\frac{2\left(\sigma_{v}-\sigma_{h}\right)}{3} \frac{\dot{\mu}}{\mu}\right] \underline{e}_{3} \otimes \underline{e}_{3} .
\end{aligned}
$$

Equating the vertical component in the above equality with 79 provides

$$
\frac{f^{v p}}{\eta}=-\frac{F_{v}^{e} \frac{\dot{\Lambda}}{\Lambda}+\left[\left(\sigma_{v}+2 \sigma_{h}\right) \bar{K}+2\left(\sigma_{v}-\sigma_{h}\right) \bar{\mu}\right] \dot{J}_{i r}}{K+\frac{2 \sqrt{3}}{3} \mu a}
$$

Observing that

$$
f^{v p}=A \sigma_{h}+B \sigma_{v}-p_{v p} \text { with }\left\{\begin{array}{l}
A=(-2+a \sqrt{3}) / 3 \\
B=(-1-a \sqrt{3}) / 3
\end{array}\right.
$$

identity 82 can be rewritten as

$$
\left(K+\frac{2 \sqrt{3}}{3} \mu a\right) \frac{A \sigma_{h}+B \sigma_{v}-p_{v p}}{\eta}=-\beta_{v}^{e} \frac{\dot{\Lambda}}{\Lambda}+\left[\left(\sigma_{v}+2 \sigma_{h}\right) \bar{K}+2\left(\sigma_{v}-\sigma_{h}\right) \bar{\mu}\right] \dot{J}_{i r}
$$

By observing that

$$
\operatorname{tr}_{\underline{\underline{d}}}^{i r}=\frac{\dot{J}_{i r}}{J_{i r}}=-\frac{f^{v p}}{\eta}=-\frac{A \sigma_{h}+B \sigma_{v}-p_{v p}}{\eta}
$$

and equating the horizontal components in 81 , one obtains 


$$
\dot{\sigma}_{h}=\beta_{h}^{e} \frac{\dot{\Lambda}}{\Lambda}-\left(K-\frac{\sqrt{3}}{3} \mu a\right) \frac{\dot{J}_{i r}}{J_{i r}}+\left[\left(\sigma_{v}+2 \sigma_{h}\right) \bar{K}-\left(\sigma_{v}-\sigma_{h}\right) \bar{\mu}\right] \dot{J}_{i r}
$$

Equations 84-86 form the nonlinear PDE system that describes the mechanical state of the elasto-viscoplastic layer during the postdeposition period. This PDE system involves only temporal derivatives due to Lagrangian description, which allows its reduction to a parameterized ODE system. It consists of an initial value problem (IVP) that governs the unknown fields $\sigma_{h}, J_{i r}$, and $\Lambda$. The system is solved with MAPLE using a numerical integration routine based on Runge-Kutta-Fehlberg method, which produces a solution accurate to the fifth order. The initial condition at time $t=T^{s}$ is defined by the fields $\sigma_{h}^{p s}\left(X_{3}\right)=\sigma_{h}\left(x_{3}=X_{3}, t=T^{s}\right), J_{i r}^{p s}\left(X_{3}\right)=J_{i r}\left(x_{3}=X_{3}, t=T^{s}\right)$, and $\Lambda^{p s}\left(X_{3}\right)=\Lambda\left(x_{3}=X_{3}\right.$, $\left.t=T^{s}\right)$ that were computed at the end of accretion period.

Once the stretch field $\Lambda\left(X_{3}, t\right)$ is calculated, the compaction law $t \rightarrow H(t)$ is then obtained from the displacement of particles located at the top of the basin. It is recalled that the displacement field results from numerical integration of following relationship:

$$
\nabla \underline{\xi} \cdot \underline{e}_{3}=\frac{\partial \xi_{3}\left(X_{3}, t\right)}{\partial X_{3}}=\Lambda\left(X_{3}, t\right)-1
$$

The gravitational compaction law is obtained by the numerical integration of the previous expression.

\section{4 | ILLUSTRATIVE NUMERICAL BASIN SIMULATIONS-COMPARISON WITH FE SOLUTIONS}

For illustrative purposes, the semianalytical solutions developed in Section 3 are presented in the context of a sedimentary basin simulation during both the accretion and postaccretion phases. The numerical results do not refer to a real data life basin but rather to an academic problem and are only intended to illustrate some features of basin deformation in the context of purely gravitational compaction under oedometric conditions. In the framework of such simplified scenario, the accuracy of the approach predictions is assessed through comparison with FE solutions derived from the basin simulator developed by Brüch et al. ${ }^{36,51}$. This simulator, which implements a finite thermo-poro-mechanics modeling, is specifically devised to deal with 3D analyses of purely mechanical and chemo-mechanical compaction in sedimentary basins. The main features of the FE constitutive modeling include elasticity coupling with large irreversible porosity changes as well as the sediment material hardening induced by irreversible densification during compaction. ${ }^{43}$

From a computational viewpoint, the basin simulator relies upon a parallel FE implementation of the constitutive modeling with shared memory multiprocessing interface. An automatic timestep algorithm allows for continuous update of the time step length used in the analyses, based on the evolution the material properties and FE mesh geometry. The issue related to the fact the basin is an open material during the sediment accretion phase is addressed by means of a specific activation/deactivation procedure. Basically, the latter consists in operating with a fictitious closed material system in which the sediment deposition periods are modeled by progressive activation of gravity forces and material properties within the fictitious basin sublayers. At each geological time $t$, the configuration of the real basin system and associated mechanical state is deduced from the evolution in time of the fictitious closed material system. Further description of the activation/deactivation algorithm and its numerical implementation may be found in the original work by Bernaud et al. ${ }^{44}$ and related subsequent extensions. ${ }^{9,51}$

\section{1 | Problem description and data}

We analyze the one-dimensional compaction induced by purely gravitational effects in a sedimentary basin that forms during a geological period $0 \leq t \leq T^{s}=60$ My by continuous material supply with a constant rate of sediment accretion equal to $\dot{M}_{d}=2.54 \times 10^{-9} \mathrm{~kg} / \mathrm{s}$ per unit area, approximately equivalent to a rate of $80 \mathrm{ton} / \mathrm{km}^{2} /$ year. In its reference state, the sediment material is assumed to exhibit always the same mechanical properties along the accretion phase. In order to characterize the level of compaction magnitude, it is relevant to note that, in the absence of any compaction process, the total amount of deposited sediments would correspond to a vertical column of thickness 


$$
\mathcal{H}=\underset{d}{M} T^{s} / \rho_{0}
$$

As regards the material properties in the reference state, the model data used for the numerical simulations are defined as follows. Initial material density $\rho_{0}=800 \mathrm{~kg} / \mathrm{m}^{3}$, initial porosity $\phi_{0}=0.72$, initial Young modulus $E_{0}=1 \mathrm{GPa}$, initial Poisson's ratio $\nu_{0}=0.33$, initial consolidation pressure for plasticity $p_{c 0}=4 \mathrm{MPa}$, plastic hardening law exponent $m_{p}=1.3$, initial consolidation pressure for viscoplasticity $p_{v p 0}=5 \mathrm{MPa}$, viscoplastic hardening law exponent $m_{v p}=1.0$, and viscosity coefficient $\eta=1 \mathrm{GPa} \cdot \mathrm{My}$. Finally, the scalar that controls the slope of the ductile part of both the plastic and viscoplastic yield surfaces is fixed to $a=1.1545$.

Although the configuration addressed herein refers to an illustrative synthetic case, the material properties are actually characteristic of sandstone-like sediments as no fluid overpressure is considered throughout the simulation. In addition, dissolution and precipitation of quartz minerals are known to play an important role in porosity reduction of siliciclastic rocks like sandstones. ${ }^{58}$ However, the choice of some parameters such as the Young modulus and the plastic consolidation pressure is questionable, as they seem unrealistic at the deposition reference state. The high value adopted for $E_{0}$ is attributed to the limitation of the Hashin-Shtrikman estimates 22 to adequately predict the evolution of the stiffness moduli over a wide range of porosity. It is therefore necessary to consider a relatively high value for initial Young modulus to obtain reasonable values at depth. As regards the initial consolidation pressure for plasticity, a deliberately high value is adopted for to better visualize the model predictions and thus clearly illustrate each phase of the compaction process described in Section 3. More realistic values of $p_{c 0}$ could actually be adopted in order to activate plastic deformation at low overburden levels (see, e.g., Brüch et al. ${ }^{52}$ ). As it will be commented in Section 4.2, the values considered for material properties in the reference state lead to unrealistically overestimating the extent of upper elastic layer. However, this superficial crust of the sedimentary basin generally represents a minor component in basin modeling, so that it is more important to relevantly characterize the rock behavior at depth.

According to selected values for the rate of sediment accretion $\dot{M}_{d}$, initial material density $\rho_{0}$, and total accretion phase duration $T^{\text {s }}$, it follows from 88 that the whole deposition process would result in a sediment column of thickness $\mathcal{H}=6000 \mathrm{~m}$, corresponding to a thickness increase of $100 \mathrm{~m}$ per million years of increase in the absence of compaction.

As regards the computational FE model, the model geometry representing the initial configuration of the fictitious material system is defined by a column with $\mathcal{H}=6000 \mathrm{~m}$ height and $L_{1}=L_{2}=100 \mathrm{~m}$ horizontal sides (Figure 9). The initial mesh of the column consists in $n=60$ twenty-node hexahedra elements (quadratic displacement interpolation) of side $L=100 \mathrm{~m}$, regularly distributed along the column height. The associated total number of nodes is equal to 728, corresponding to 2,184 degrees of freedom (nodal displacement components). Each element represents in fact a sublayer that shall be activated according to its location as sediment accretion proceeds.

The boundary conditions for the FE model are summarized in Figure 9 and are consistent with the oedometric assumption adopted for the analysis. The plane $x_{3}=0$ defines the rigid basement rock, thus implying the displacement condition $\underline{\xi}\left(x_{3}=0, t\right) \cdot \underline{e}_{3}=0$. The normal displacements along lateral surfaces $x_{i}=0$ and $x_{i}=L_{i}(i=1,2)$ are maintained null: $\underline{\xi}\left(x_{i}=0, t\right) \cdot \underline{e}_{i}=\underline{\xi}\left(x_{i}=L_{i}, t\right) \cdot \underline{e}_{i}=0$. During all the numerical simulations, the upper surface $x_{3}=H(t)$ remains stress free: $\underline{T}=\underline{\underline{\sigma}}\left(x_{3}=H(t), t\right) \cdot \underline{e}_{3}=\underline{0}$.

Finally, the total time of basin evolution analysis is fixed to $T^{f}=2 \times T^{s}=120 \mathrm{My}$, and the initial time step is taken equal to $\Delta t=0.01 \mathrm{My}$, which is much smaller than the characteristic viscoplastic relaxation time $\mathcal{T}_{c}^{v p}=\eta / E_{\text {oed }} \simeq$ $0.675 \mathrm{My},{ }^{59}$ where $E_{\text {oed }}$ denotes the initial oedometric elastic modulus.

\section{2 | Results}

For the sake of clarity, the results of the present analysis will be referred to as follows. In all figures, the semianalytical solutions are referred to as "SAR" and displayed by solid continuous lines, whereas the results derived from the FE basin simulator are referred to as "FEM" and represented by symbols.

Prior to results analysis, it is recalled once again that the numerical simulation is carried out in the context of an academic situation, and the associated results are provided for merely illustrative purposes. They are not intended to reproduce the mechanical fields that could be observed in real data life basins.

At the scale of basin, a main feature in sedimentary basin simulation refers to assessment of the compaction law $t \rightarrow H(t)$. The semianalytical and FE predictions obtained for the gravitational compaction law are shown in Figure 10. The different phases related to basin behavior that have been described in Section 3 are highlighted in this figure. The 


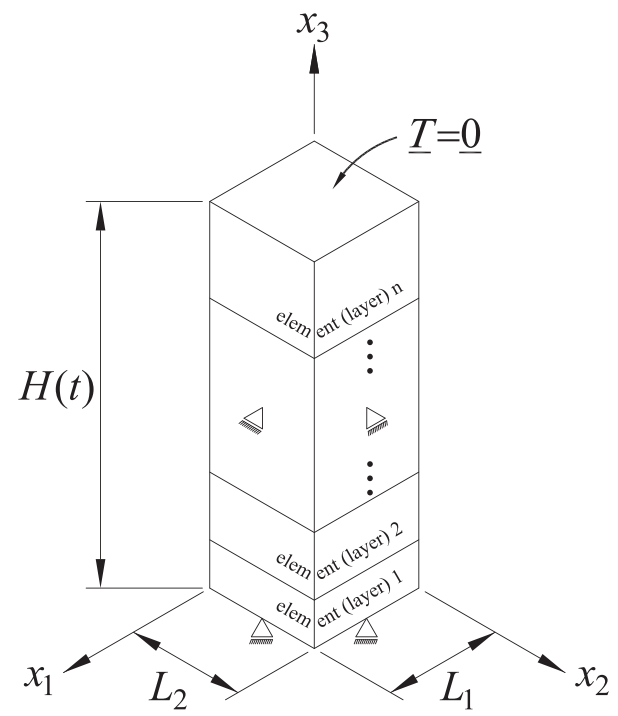

F I G U RE 9 Spatial discretization and mechanical boundary conditions

SAR predictions are very close to the FEM solutions, and this good agreement can thus be viewed as a preliminary validation of the simplifying assumption introduced in Section 3.1.4 (elastic-viscoplastic phase). The level of basin compaction can be defined at time $t$ as $1-H(t) / \mathcal{H}$. The magnitudes of this compaction ratio predicted by SAR and FEM at $t=T^{s}$ (end of the accretion phase) are respectively $52.2 \%$ and 52.3\%. In the postaccretion period, the compaction level exhibits a slight decrease to reach approximately 54\% for both SAR and FEM at $t=T^{f}$ (end of the analysis). The thicknesses of the different layers and the associated times of formation during the deposition period obtained from the semianalytical approach are summarized in Table 1.

\subsection{1 | Numerical analysis of local fields}

This section is dedicated to analyzing the evolution of mechanical fields attached to the sediment particles located at any instant along the substratum interface $x_{3}=0$ (i.e., bottom layer). These particles are deposited at the beginning $t=0$ of accretion process and associated mechanical state is evolving as burial proceeds, exhibiting the highest strain

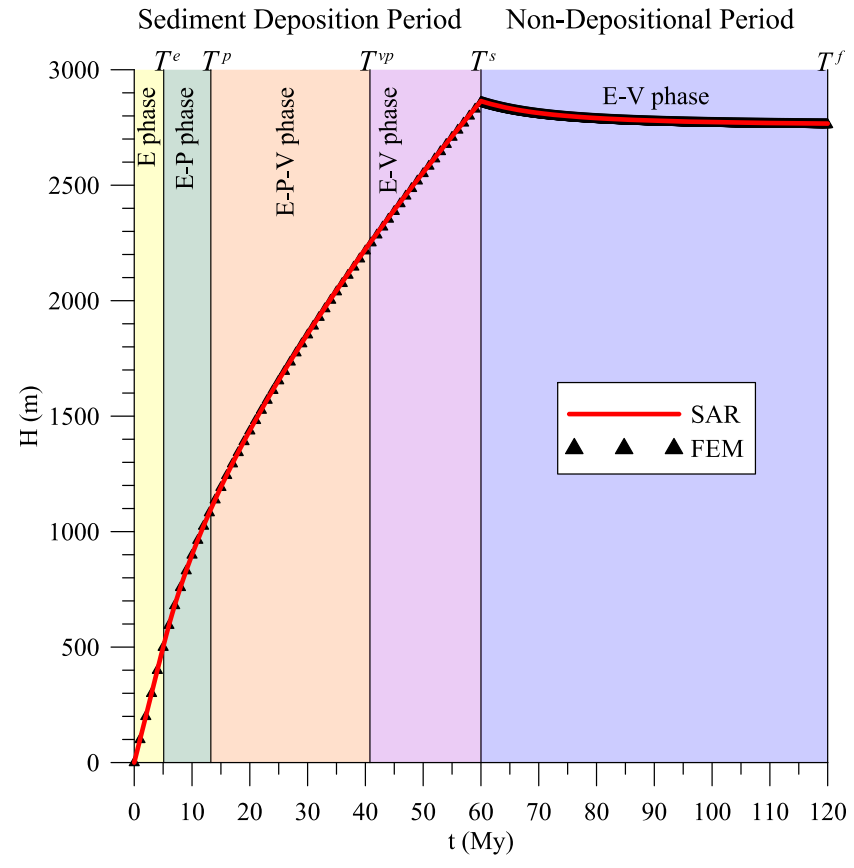

F I G URE 10 Gravitational compaction law of the basin [Colour figure can be viewed at wileyonlinelibrary.com] 
TA B LE 1 Time of formation and thickness of each layer predicted from SAR

\begin{tabular}{lrrr}
$T^{e}$ & $5.10 \mathrm{My}$ & $H^{e}$ & $509.20 \mathrm{~m}$ \\
$T^{p}$ & $13.26 \mathrm{My}$ & $H^{p}$ & $1097.37 \mathrm{~m}$ \\
$T^{v p}$ & $40.79 \mathrm{My}$ & $H^{v p}$ & $2249.96 \mathrm{~m}$ \\
$T^{s}$ & $60.00 \mathrm{My}$ & $H^{s}$ & $2864.21 \mathrm{~m}$ \\
\hline
\end{tabular}

Abbreviation: SAR, semianalytical solution.

and stress levels of the basin. Table 2 summarizes the values of relevant quantities predicted by both SAR and FEM analyses at particular times $t=T^{s}$ and $t=T^{f}$.

The evolution of Eulerian porosity $\varphi\left(x_{3}=0, t\right)$ and mass density $\rho\left(x_{3}=0, t\right)$ are respectively depicted in Figure 11A and B. As expected, the porosity decrease with time during the accretion period is straightforwardly associated with continuous increase in mass density of the porous material. These quantities rapidly reach almost stabilized values as soon as sediment deposition process stops.

Once again, the perfect matching between SAR and FEM predictions is emphasizing the accuracy of the semianalytical approach. The small discrepancy observed in the elastic-viscoplastic phase is reflecting the effect of the approximation introduced in Section 3.1.4 in the formulation of problem governing equations.

The evolution of stress state along accretion and postaccretion phase is displayed in Figure 12A. As expected from equilibrium consideration described in 79 , the vertical stress component is no more evolving after the deposition process has stopped $t \geq T^{s}$, whereas the horizontal component progressively stabilizes. The evolution in time of the different components of the Jacobian of the particle transformation is shown in Figure 12B. It is first observed that the values of total Jacobian $J$ and its irreversible counterpart $J_{i r}$ are revealed to be very close, thus validating the assumption of infinitesimal elastic strains adopted in the analysis. It is recalled that the plastic part $J_{p}$ (resp. viscoplastic part $J_{v p}$ ) represents a measure of plastic (resp. viscoplastic) volumetric dilatation undergone by the sediment material. This figures provides a clear illustration that plastic compaction is prevailing for $t \leq T^{p}$ where the plastic part $J_{p}$ of the Jacobian is significantly decreasing as burial proceeds to finally stabilize after the elastic-viscoplastic phase (along which $\underline{\underline{d}}^{p}=0$ and thus $\dot{J}_{p}=0$ ) is activated at $t=T^{v p}$. The evolution of viscoplastic component $J_{v p}$ exhibits an opposite trend: it starts decreasing from unity at time $t=T^{p}$ with simultaneous evolution of plastic and viscoplastic strains until $t=T^{v p}$, and from that moment and on, only the viscoplastic volumetric dilatation continues evolving $\dot{J}_{v p}<0$.

Finally, the hardening of the material induced by large porosity change is illustrated in Figure 13 by plotting the plastic $p_{c}$ and viscoplastic $p_{v p}$ consolidation pressures as functions of time. The results in this figure indicate that material densification may lead to significant increase in the value of hardening parameter, which can be multiplied by a factor as high as 10 .

T A B LE 2 Relevant mechanical quantities evaluated for particles along $x_{3}=0$

\begin{tabular}{|c|c|c|c|c|}
\hline & \multicolumn{2}{|c|}{$t=T^{s}=60 \mathrm{My}$} & \multicolumn{2}{|c|}{$t=T^{f}=120 \mathrm{My}$} \\
\hline & SAR & FEM & SAR & FEM \\
\hline$\rho\left(\mathrm{kg} / \mathrm{m}^{3}\right)$ & 2654.44 & 2671.70 & 2732.32 & 2722.86 \\
\hline$\sigma_{h}(\mathrm{MPa})$ & -39.32 & -39.13 & -40.46 & -40.05 \\
\hline$J_{i r}$ & 0.3020 & 0.2995 & 0.2932 & 0.2938 \\
\hline$J_{p}$ & 0.3821 & 0.3803 & 0.3821 & 0.3803 \\
\hline$J_{v p}$ & 0.7904 & 0.7873 & 0.7675 & 0.7725 \\
\hline$p_{c}(\mathrm{MPa})$ & 59.48 & 62.89 & 73.95 & 72.71 \\
\hline
\end{tabular}

Abbreviation: FEM, FE basin simulator; SAR, semianalytical solution. 


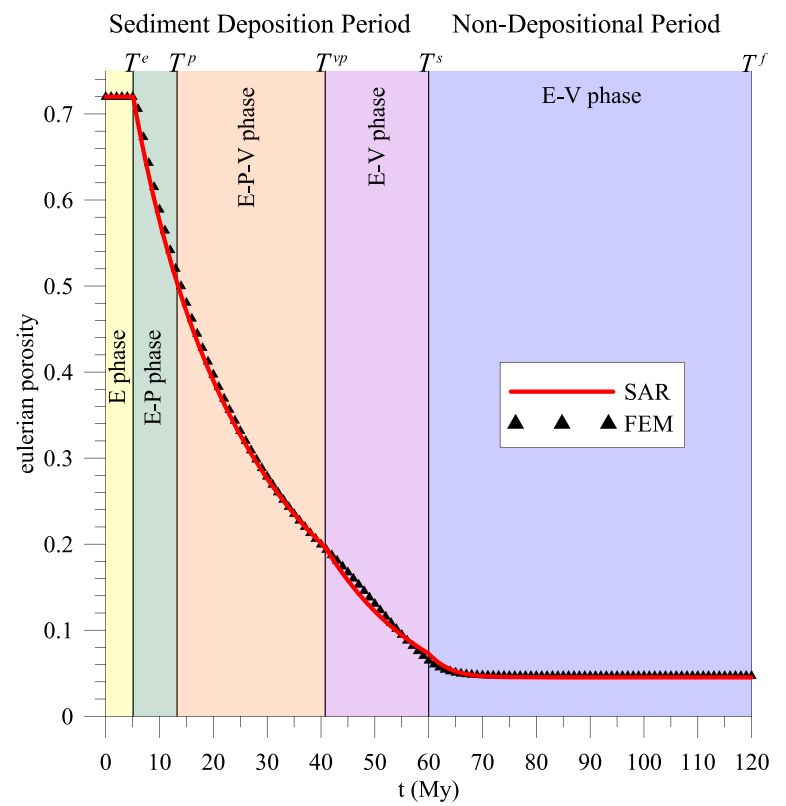

(A)

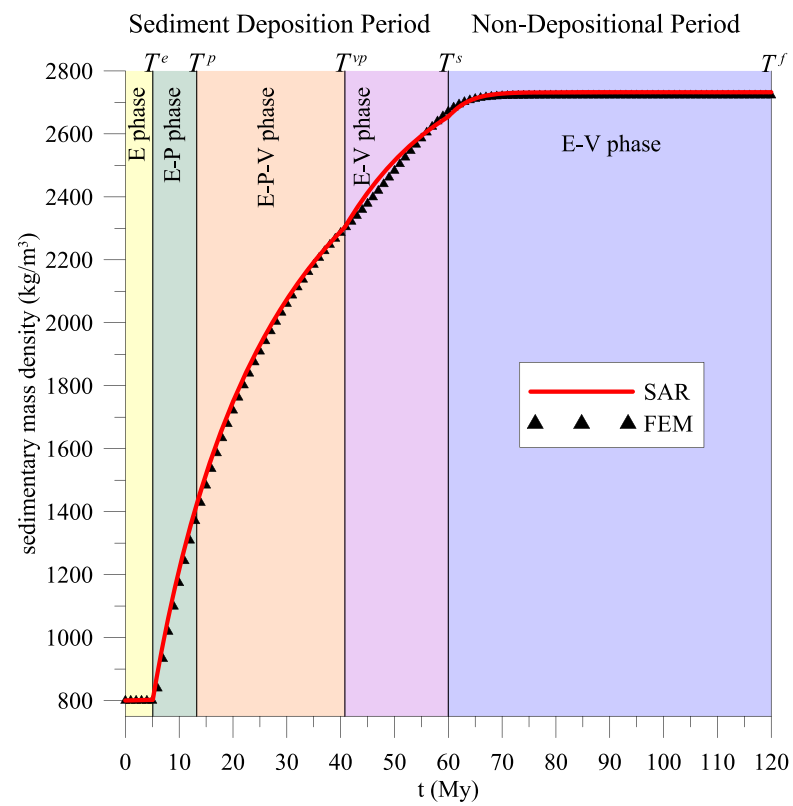

(B)

F I G U R E 11 Evolution at $x_{3}=0$ : A, Eulerian porosity; B, sedimentary mass density [Colour figure can be viewed at wileyonlinelibrary. com]

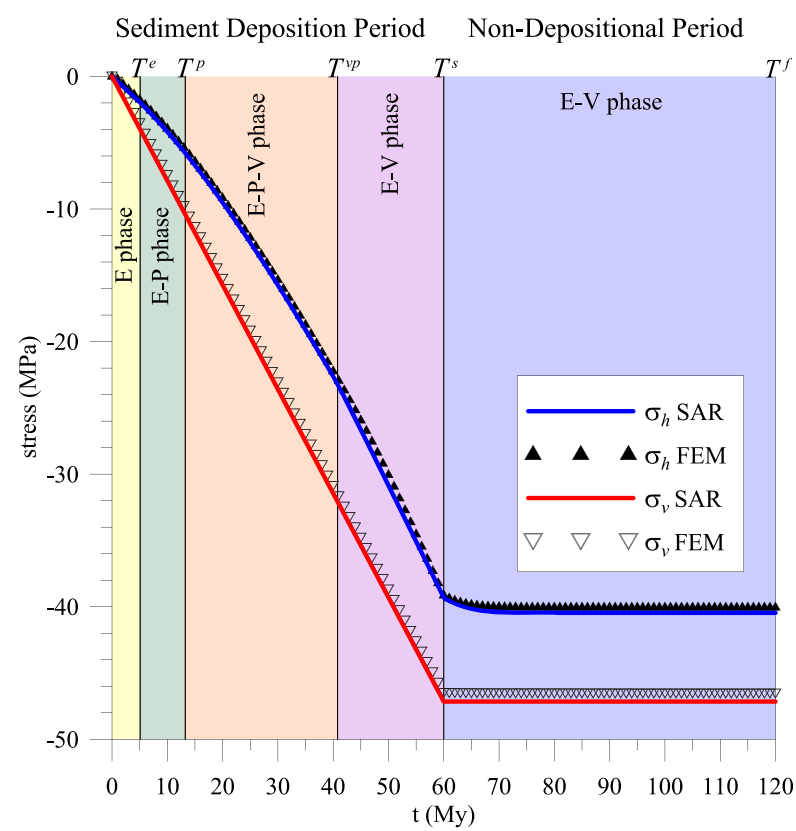

(A)

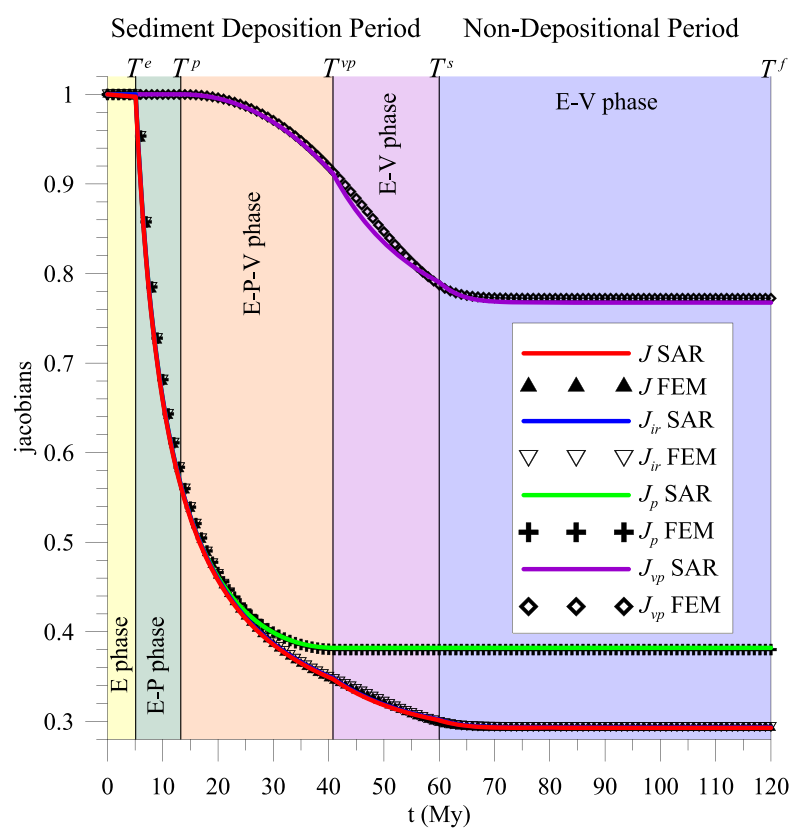

(B)

F I G U R E 12 Evolution at $x_{3}=$ 0: A, horizontal and vertical stress; B, Jacobians [Colour figure can be viewed at wileyonlinelibrary.com]

\subsection{2 | Analysis of the overall basin response}

We move in this section to the analysis of the mechanical fields that characterize the overall response of the sedimentary basin, focusing the description on the state of the latter at particular times $t=T^{s}$ and $t=T^{f}$.

The overall response is first illustrated by Figures 14-16, displaying respectively the profiles within the basin of porosity, sediment mass density, and Jacobian of the particle transformation. The analysis of the results calls for the following qualitative comments: 
F I G U RE 13 Evolution of the plastic and viscoplastic consolidation pressure at $x_{3}=0$ [Colour figure can be viewed at wileyonlinelibrary.com]

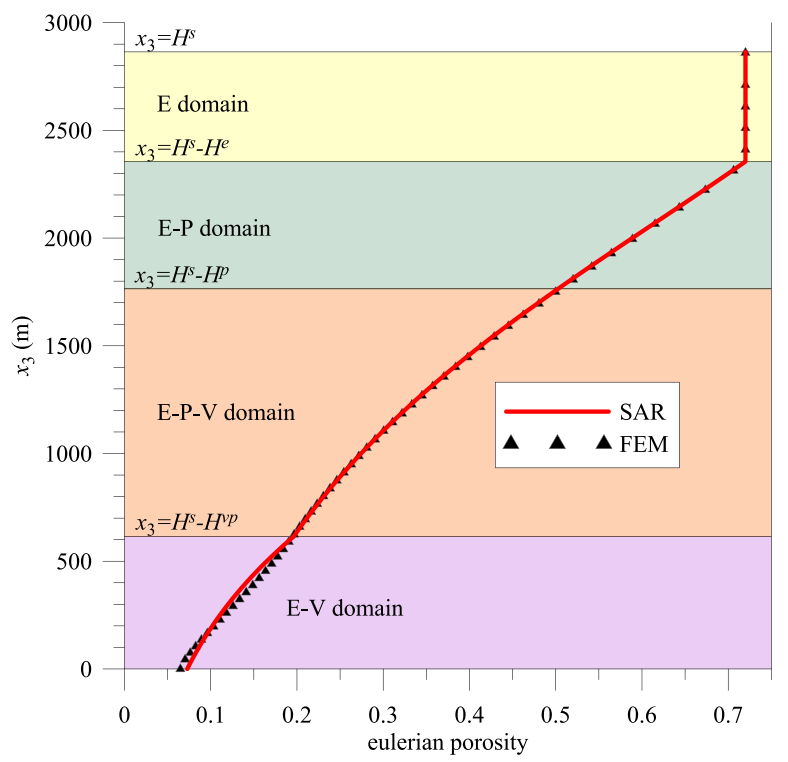

(A)
Sediment Deposition Period Non-Depositional Period
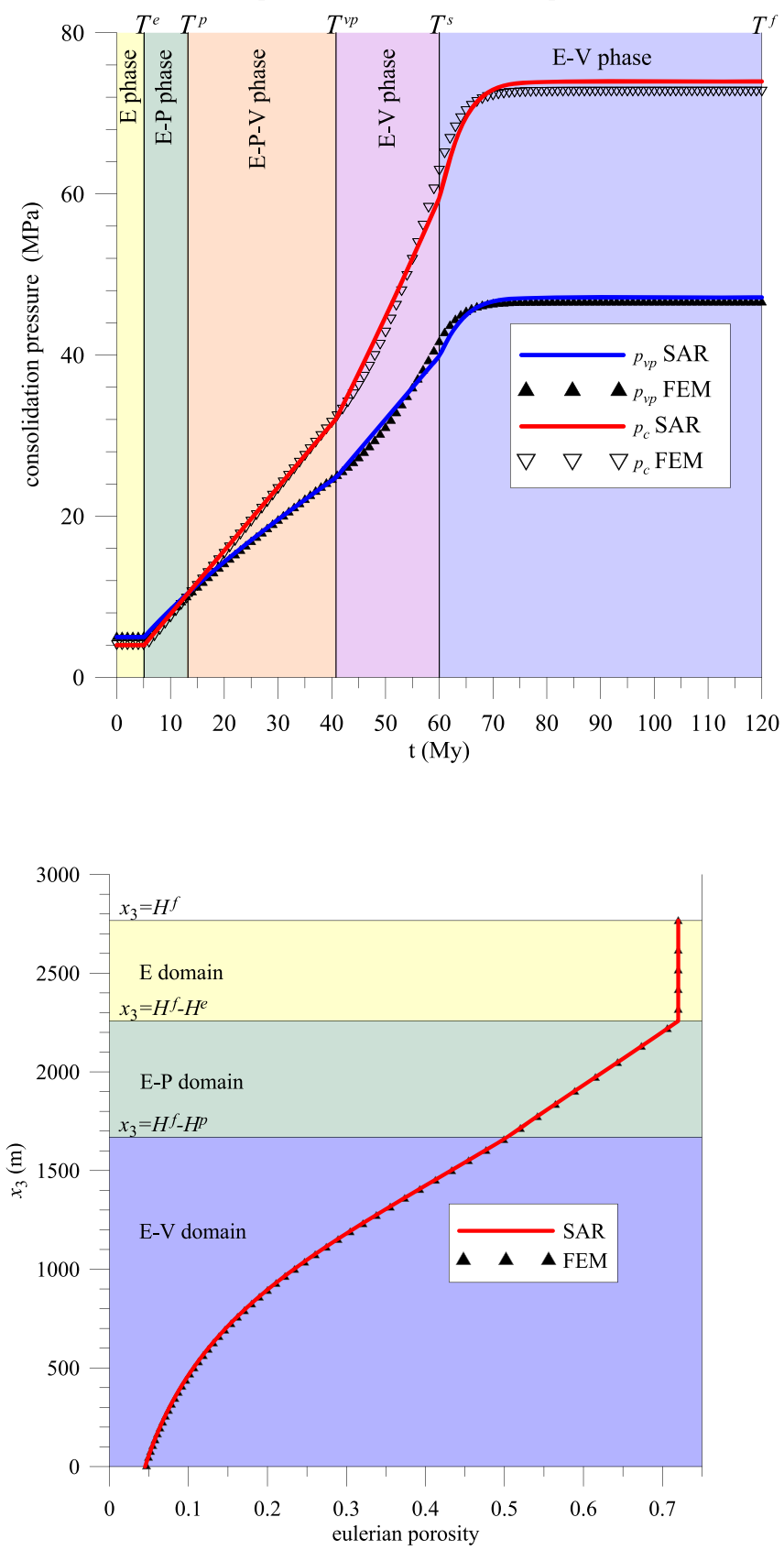

(B)

F I G U RE 14 Eulerian porosity profile along basin thickness: A, at $t=60 \mathrm{My}$; B, at $t=120 \mathrm{My}$ [Colour figure can be viewed at wileyonlinelibrary.com]

- The material densification induced by compaction at large strains is reflected by the decrease (resp. increase) of porosity (resp. mass density) with depth.

- The referred fields slightly vary along the upper elastic crust, which is consistent with the assumption of infinitesimal elastic strains adopted in the analysis. The thickness of elastic crust may appear as excessively high in regards the total thickness of the basin. This is mainly attributed to the value of $p_{c 0}$ defining the extent of elastic layer through condition $f^{p}\left(\underline{\underline{\sigma}}, p_{c 0}\right)=0$. From a rigorous point of view, $p_{c 0}$ should therefore refer to an elastic limit and not to a limit pressure state as expressed by expression 29. Consequently, such a definition of $p_{c 0}$ leads to overestimating the extent of elastic layer, as described by Deudé et al. ${ }^{43}$ 


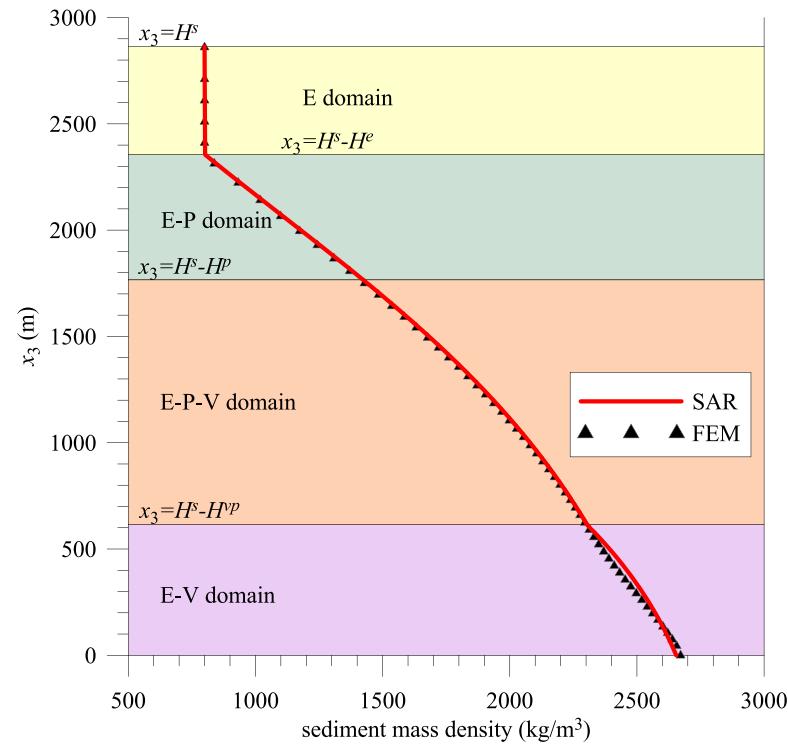

(A)

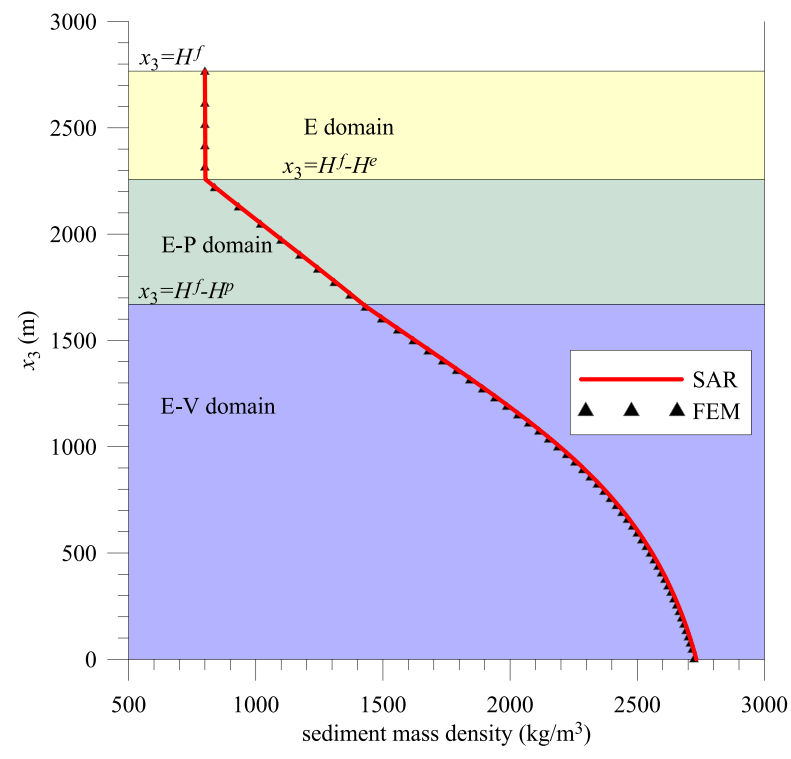

(B)

F I G U R E 15 Sediment mass density profile: A, at $t=60 \mathrm{My}$; B, at $t=120 \mathrm{My}$ [Colour figure can be viewed at wileyonlinelibrary.com]

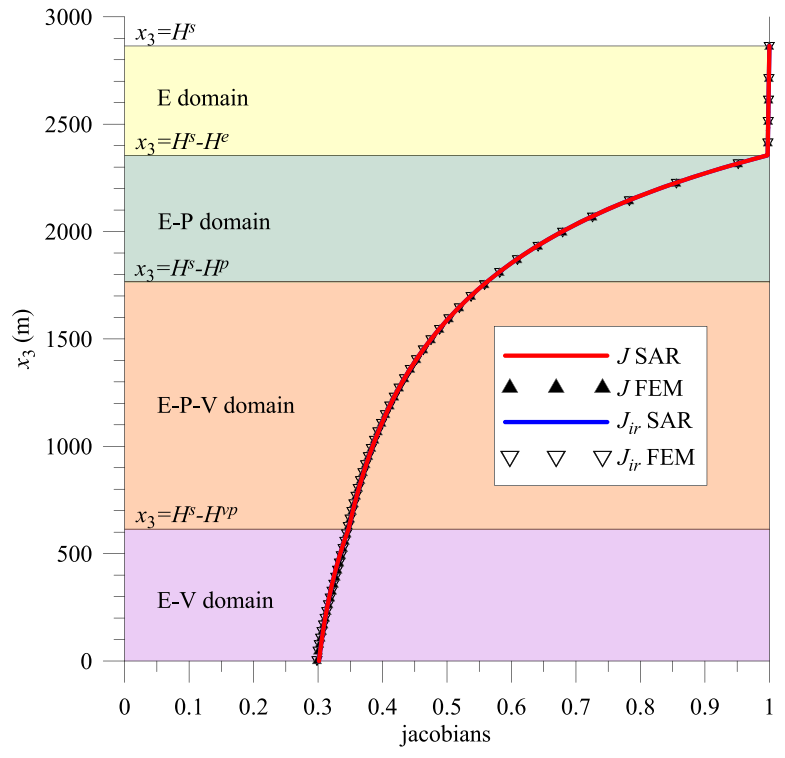

(A)

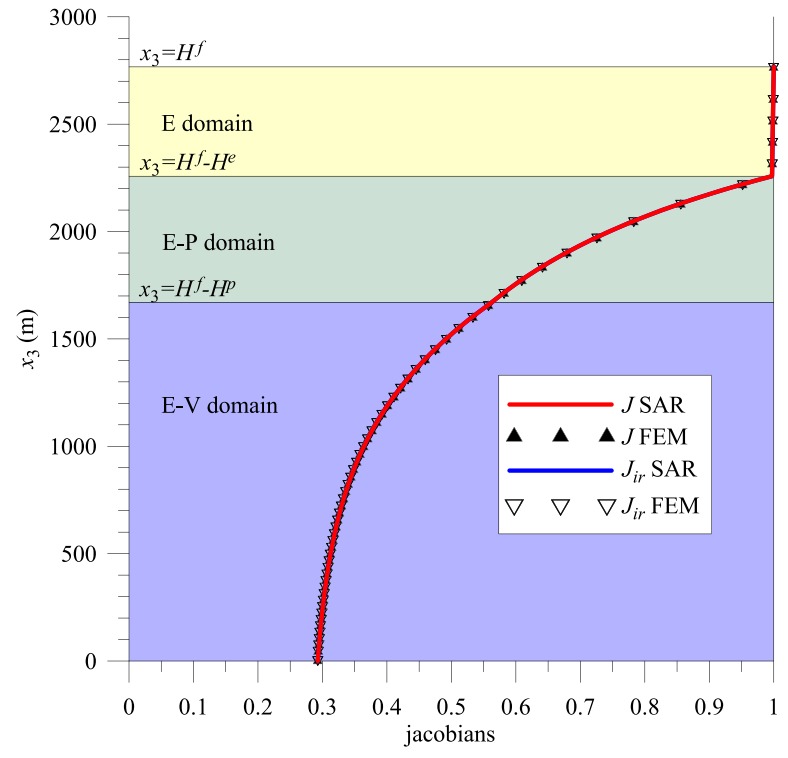

(B)

F I G U R E 16 Jacobians of the transformation profiles: A, at $t=60 \mathrm{My}$; B, at $t=120 \mathrm{My}$ [Colour figure can be viewed at wileyonlinelibrary.com]

- The two approaches SAR and FEM predict similar profiles of all fields for both $t=T^{\mathrm{s}}$ and $t=T^{f}$. The small discrepancy observed in the elasto-viscoplastic layer located at the bottom of the basin is due to the simplifying approximation introduced in Section 3.1.4 for the formulation of governing equations.

The profiles of horizontal and vertical stresses within the basin are plotted in Figure 17. It is recalled that the vertical stress component $\sigma_{v}$ is statically determined independently on the material constitutive behavior. In contrast, the horizontal stress component $\sigma_{h}$ is strongly affected by the constitutive properties of sediment material. In particular, it is very sensitive to the value of parameter $a$ controlling the inclination of plastic/viscoplastic yield surface in the contractive stress states. 


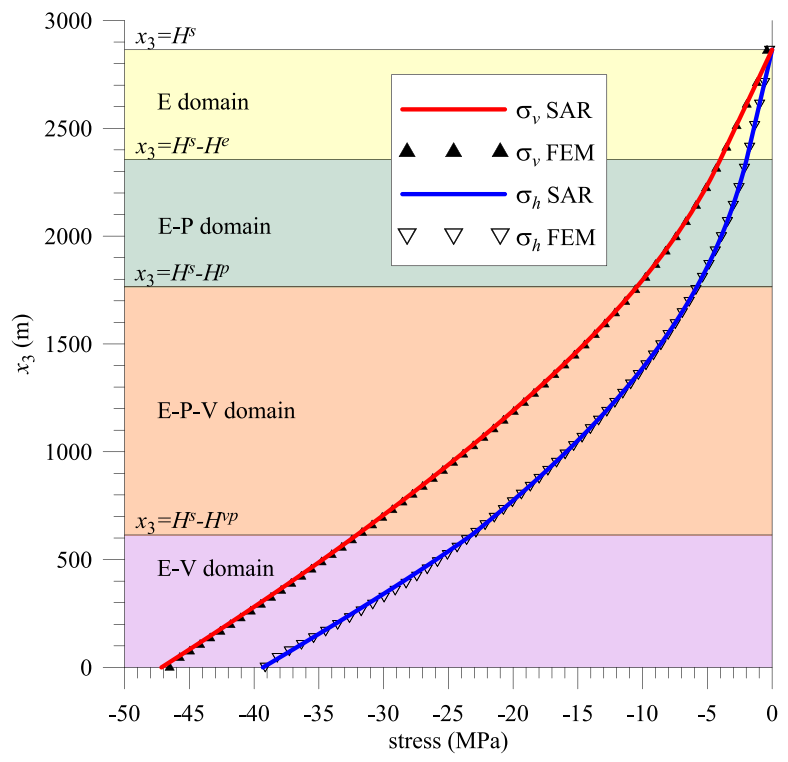

(A)

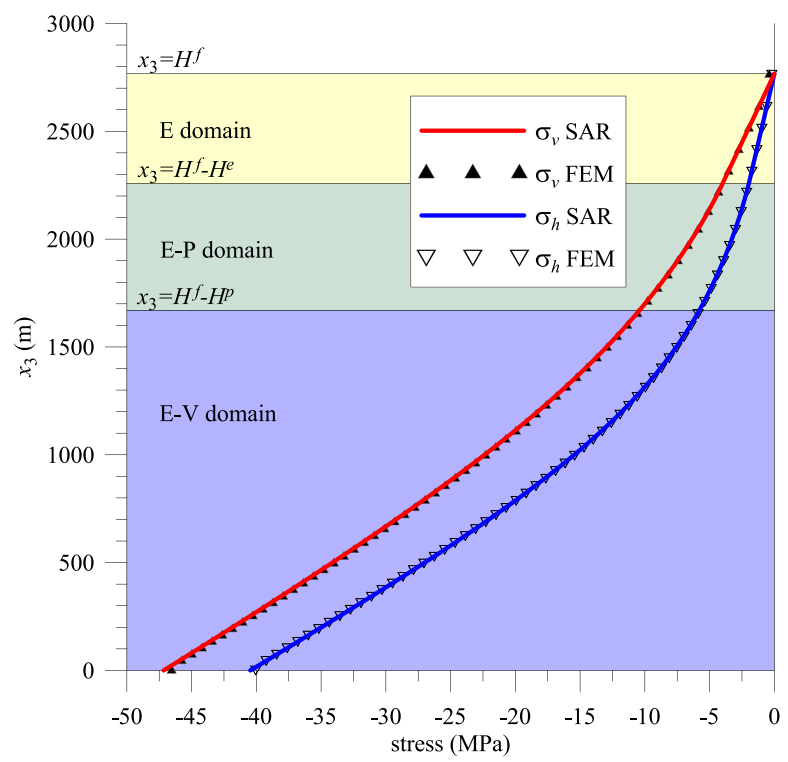

(B)

F I G U RE 17 Stress profiles within the basin: A, at $t=60 \mathrm{My}$; B, at $t=120 \mathrm{My}$ [Colour figure can be viewed at wileyonlinelibrary.com]

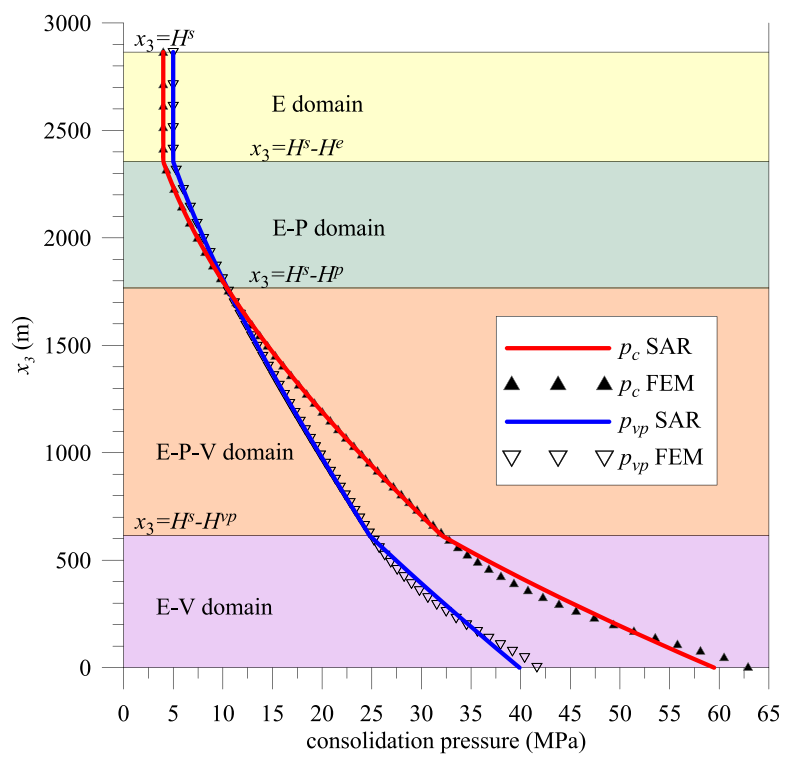

(A)

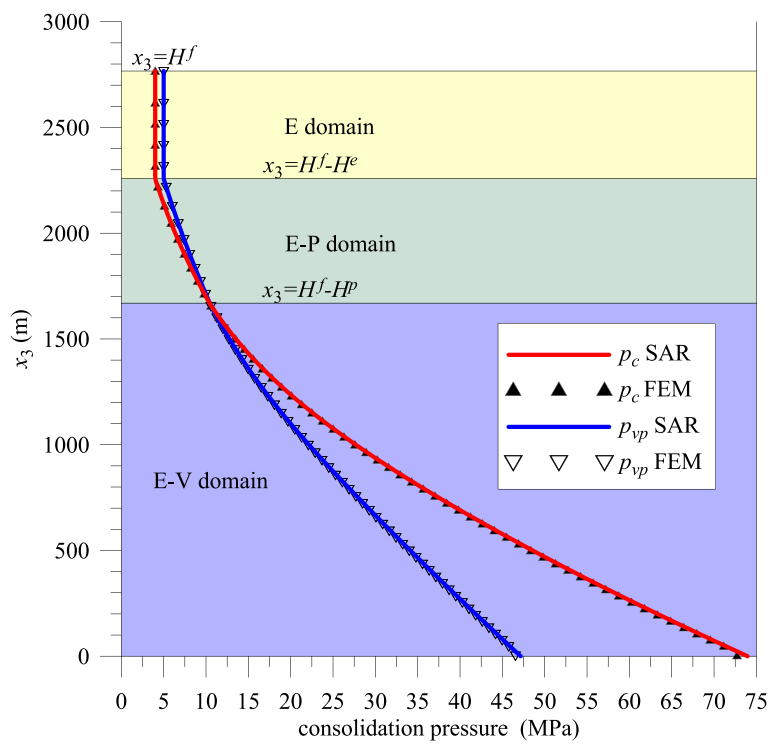

(B)

FI G U RE 18 Profiles of plastic and viscoplastic consolidation pressures: A, at $t=60 \mathrm{My}$; B, at $t=120 \mathrm{My}$ [Colour figure can be viewed at wileyonlinelibrary.com]

Finally, the plastic and viscoplastic hardening parameter profiles in the basin are shown in Figure 18, illustrating once again the hardening of material induced by densification.

\section{5 | CONCLUSIONS}

A theoretical framework for the analysis of gravitational compaction in sedimentary basins under oedometric conditions at large strains has been formulated. The latter relies upon a simplified description of the problem geometry and geological process, as well as of sediment material constitutive behavior. In the context, the field 
equations governing the evolution of mechanical state of the sedimentary basin have carefully described and the semianalytical solutions to associated nonlinear PDE system have been derived making use of the MAPLE software recourses. These solutions prove useful in the context of sedimentary basins modeling because they can be viewed as reference solutions for verification and benchmarks of basin simulators. As a matter of fact, there is a lack for reference solutions relevant to the field of basin simulation that incorporate essential features of sediment deformation induced by compaction processes. In that respect, particular emphasis has been dedicated for the constitutive modeling formulated at porous material level to take into account the stiffening and hardening associated with pore closure.

From the constitutive modeling viewpoint, the state equations of the sediment material are formulated in the framework of coupled plasticity-viscoplasticity at large strains. At macroscopic scale, the purely mechanical compaction that predominates in the upper layers of a sedimentary basin is modeled by means of time-independent plastic strains, whereas the viscoplastic component of behavior is intended to address creep-like deformation resulting in chemo-mechanical compaction that prevails at deeper layers. The proposed coupled modeling is able to automatically account, through the irreversible component of the material transformation Jacobian, for deformation along intermediate layers where the two compaction processes occur simultaneously. A main advantage of adopting the simplified oedometric setting is to allow for investigation and clear interpretation of the role of each relevant model property on the mechanical evolution of the sedimentary basin.

Even though it mainly refers to an academic problem rather than to real data life basin, the numerical illustration proved able to accurately capture fundamental features of basin deformation in both accretion and postdepositional periods of the geological basin life. The numerical simulations have addressed the overall basin response, such as the prediction of compaction law, stresses and porosity profiles within the basin, and the evolution in time of the relevant mechanical parameters at the bottom of the basin. The proposed approach can also be used for intensive parametric analyses, because it does not require complex and costly computational procedures such as those involved in FE simulations.

Comparison with FE solutions derived from a basin simulator that integrates the effects of large irreversible porosity change on the elastic properties as well as on plastic/viscoplastic hardening laws ${ }^{36,51}$ provides ample evidence of the ability of derived semianalytical solutions to relevantly reflect most of local and overall features of the basin deformation. In particular, it has been shown that the simplifying approximations introduced in the resolution formulation affect very little the accuracy of obtained predictions.

As extensions to be foreseen in the future, the analysis should be extended to more realistic situations incorporating (a) the effects of tectonic loading by applying for instance lateral prescribed displacements, ${ }^{9}$ (b) the effects of geothermal field relying on the approach developed in Brüch et al. ${ }^{36}$ to address the evolution of sediment material properties associated with temperature, and (c) hydromechanical coupling.

\section{ORCID}

Paulo Sérgio B. Lemos (D) https://orcid.org/0000-0002-1614-6544

André Brüch (10) https://orcid.org/0000-0002-5168-1470

Samir Maghous (1) https://orcid.org/0000-0002-1123-3411

\section{REFERENCES}

1. Schmidt V, Mcdonald DA. The role of secondary porosity in the course of sandstone diagenesis. In: Scholle PA, Schluger PR, eds. Aspects of Diagenesis. Vol.26; 1979:175-207.

2. Athy LF. Density, porosity, and compaction of sedimentary rocks. Am Assoc Pet Geol Bull. 1930;14(1):1-24. https://doi.org/10.1306/ 3d93289e-16b1-11d7-8645000102c1865d

3. Hubbert MK, Rubey WW. Role of fluid pressure in mechanics of overthrust faulting: I mechanics of fluid-filled porous solids and its application to overthrust faulting. Bull Geol Soc Am. 1959;70(2):115-166. https://doi.org/10.1130/0016-7606(1959)70

4. Smith JE. The dynamics of shale compaction and evolution of pore-fluid pressures. J Int Assoc Math Geol. 1971;3(3):239-263. https://doi. org/10.1007/BF02045794

5. Schneider F, Potdevin JL, Wolf S, Faille I. Mechanical and chemical compaction model for sedimentary basin simulators. Tectonophysics. 1996;263(1-4):307-317. https://doi.org/10.1016/S0040-1951(96)00027-3

6. Gutierrez M, Wangen M. Modeling of compaction and overpressuring in sedimentary basins. Mar Pet Geol. 2005;22(3):351-363. https:// doi.org/10.1016/j.marpetgeo.2005.01.003

7. Buiter SJH, Pfiffner OA, Beaumont C. Inversion of extensional sedimentary basins: a numerical evaluation of the localisation of shortening. Earth Planet Sci Lett. 2009;288(3):492-504. https://doi.org/10.1016/j.epsl.2009.10.011 
8. Jarosinski M, Beekman F, Matenco L, Cloetingh S. Mechanics of basin inversion: finite element modelling of the Pannonian Basin System. Tectonophysics. 2011;502(1-2):121-145. https://doi.org/10.1016/j.tecto.2009.09.015

9. Maghous S, Brüch A, Bernaud D, Dormieux L, Braun AL. Two-dimensional finite element analysis of gravitational and lateral-driven deformation in sedimentary basins. Int J Numer Anal Methods Geomech. 2014;38(7):725-746. https://doi.org/10.1002/nag.2232

10. Albertz M, Sanz PF. Critical state finite element models of contractional fault-related folding: part 2. Mechanical analysis. Tectonophysics. 2012;576-577:150-170. https://doi.org/10.1016/j.tecto.2012.06.016

11. Crook AJL, Willson SM, Yu JG, Owen DRJ. Predictive modelling of structure evolution in sandbox experiments. J Struct Geol. 2006; 28(5):729-744. https://doi.org/10.1016/j.jsg.2006.02.002

12. Miranda PAMN, Vargas EA, Moraes A. Evaluation of the modified Cam Clay model in basin and petroleum system modeling (BPSM) loading conditions. Mar Pet Geol. 2020;112:104-112. https://doi.org/10.1016/j.marpetgeo.2019.104112

13. Guy N, Colombo D, Frey J, Cornu T, Cacas-Stentz MC. Coupled modeling of sedimentary basin and geomechanics: a modified DruckerPrager Cap model to describe rock compaction in tectonic context. Rock Mech Rock Eng. 2019;52(10):3627-3643. https://doi.org/10.1007/ s00603-019-01783-y

14. Obradors-Prats J, Rouainia M, Aplin AC, Crook AJL. Assessing the implications of tectonic compaction on pore pressure using a coupled geomechanical approach. Mar Pet Geol. 2017;79:31-43. https://doi.org/10.1016/j.marpetgeo.2016.10.017

15. Bouziat A, Guy N, Frey J, et al. An assessment of stress states in passive margin sediments: iterative hydro-mechanical simulations on basin models and implications for rock failure predictions. Geosci. 2019;9(11):469-491. https://doi.org/10.3390/geosciences9110469

16. Barnichon JD, Charlier R. Finite element modelling of the competition between shear bands in the early stages of thrusting: strain localization analysis and constitutive law influence. Geol Soc Spec Pub. 1996;99(1):235-250. https://doi.org/10.1144/GSL.SP.1996.099.01.18

17. Dewers T, Hajash A. Rate laws for water-assisted compaction and stress-induced water-rock interaction in sandstones. $J$ Geophys Res. 1995;100(B7):13093-13112. https://doi.org/10.1029/95jb00912

18. Dove PM. The dissolution kinetics of quartz in aqueous mixed cation solutions. Geochim Cosmochim Acta. 1999;63(22):3715-3727. https://doi.org/10.1016/S0016-7037(99)00218-5

19. Gratier JP, Guiguet IR. Experimental pressure solution-deposition on quartz grains: the crucial effect of the nature of the fluid. $J$ Struct Geol. 1986;8(8):845-856. https://doi.org/10.1016/0191-8141(86)90030-1

20. Gundersen E, Renard F, Dysthe DK, Bjørlykke K, Jamtveit B. Coupling between pressure solution creep and diffusive mass transport in porous rocks. J Geophys Res Solid Earth. 2002;107(B11):2317-ECV 19-19. https://doi.org/10.1029/2001jb000287

21. Lehner FK. A model for intergranular pressure solution in open systems. Tectonophysics. 1995;245(3-4):153-170. https://doi.org/10.1016/ 0040-1951(94)00232-X

22. Neveux L, Grgic D, Carpentier C, Pironon J, Girard JP. Influence of hydrocarbon injection on the compaction by pressure-solution of a carbonate rock: an experimental study under triaxial stresses. Mar Pet Geol. 2014;55:282-294. https://doi.org/10.1016/j.marpetgeo.2014. 01.012

23. Rutter EH, Elliott D. The kinetics of rock deformation by pressure solution. Philos Trans R Soc A Math Phys Eng Sci. 1976;283(1312): 203-217. https://doi.org/10.1098/rsta.1976.0079

24. Schutjens PMTM, Spiers CJ. Intergranular pressure solution in NaCl: grain-to-grain contact experiments under the optical microscope. Rev l'Institut Fr du Pet. 1999;54(6):729-750. https://doi.org/10.2516/ogst:1999062

25. Shimizu I. Kinetics of pressure solution creep in quartz: theoretical considerations. Tectonophysics. 1995;245(3-4):121-134. https://doi. org/10.1016/0040-1951(94)00230-7

26. Zhang X, Spiers CJ. Compaction of granular calcite by pressure solution at room temperature and effects of pore fluid chemistry. Int $J$ Rock Mech Min Sci. 2005;42(7-8):871-1102. https://doi.org/10.1016/j.ijrmms.2005.05.017

27. de Boer RB, Nagtegaal PJC, Duyvis EM. Pressure solution experiments on quartz sand. Geochim Cosmochim Acta. 1977;41(2):257-260. https://doi.org/10.1016/0016-7037(77)90233-2

28. Ehrenberg SN. Preservation of anomalously high porosity in deeply buried sandstones by grain-coating chlorite: examples from the Norwegian continental shelf. Am Assoc Pet Geol Bull. 1993;77(7):1260-1286. https://doi.org/10.1306/f4c8e062-1712-11d7-8645000102c1865d

29. Mullis AM. Determination of the rate-limiting mechanism for quartz pressure dissolution. Geochim Cosmochim Acta. 1993;57(7):14991503. https://doi.org/10.1016/0016-7037(93)90009-L

30. Worden RH, Morad S. Quartz cementation in oil field sandstones: a review of the key controversies. Quartz Cem Sandstones. 2009;1-20. https://doi.org/10.1002/9781444304237.ch1

31. Broichhausen H, Littke R, Hantschel T. Mudstone compaction and its influence on overpressure generation, elucidated by a 3D case study in the North Sea. Int J Earth Sci. 2005;94(5-6):956-978. https://doi.org/10.1007/s00531-005-0014-1

32. Obradors-Prats J, Rouainia M, Aplin AC, Crook AJL. A diagenesis model for geomechanical simulations: formulation and implications for pore pressure and development of geological structures. J Geophys Res Solid Earth. 2019;124(5):4452-4472. https://doi.org/10.1029/ 2018JB016673

33. Oelkers EH, Bjørkum PA, Murphy WM. A petrographic and computational investigation of quartz cementation and porosity reduction in North Sea sandstones. Am J Sci. 1996;296(4):420-452. https://doi.org/10.2475/ajs.296.4.420

34. Walderhaug O. Kinetic modeling of quartz cementation and porosity loss in deeply buried sandstone reservoirs. Am Assoc Pet Geol Bull. 1996;80(5):731-745.

35. Yang XS. A unified approach to mechanical compaction, pressure solution, mineral reactions and the temperature distribution in hydrocarbon basins. Tectonophysics. 2001;330(1-2):141-151. https://doi.org/10.1016/S0040-1951(00)00226-2 
36. Brüch A, Maghous S, Ribeiro FLB, Dormieux L. A thermo-poro-mechanical constitutive and numerical model for deformation in sedimentary basins. J Petrol Sci Eng. 2018;160:313-326. https://doi.org/10.1016/j.petrol.2017.10.036

37. Bourgeois E, De Buhan P, Dormieux L. Formulation d'une loi élastoplastique pour un milieu poreux saturé en transformation finie. Comptes rendus l'Académie des Sci Série II, Mécanique, Phys Chim Astron. 1995;321(5):175-182.

38. Bourgeois E, Dormieux L. Prise en compte des non-linéarités géométriques dans la modélisation de la compaction des sédiments. Rev l'Institute Fr du Pet. 1997;52(1):23-34. https://doi.org/10.2516/ogst:1997002

39. Dormieux L, Maghous S. Poroelasticity and poroplasticity at large strains. Rev l'Institut Fr du Pet. 1999;54(6):773-784. https://doi.org/10. 2516/ogst:1999065

40. Dormieux L, Maghous S. Évolution des propriétés élastiques en poroplasticité finie. Comptes Rendus l'Académie des Sci - Ser IIB - Mech. 2000;328(8):593-600. https://doi.org/10.1016/S1620-7742(00)00014-3

41. Bernaud D, Deudé V, Dormieux L, Maghous S, Schmitt DP. Evolution of elastic properties in finite poroplasticity and finite element analysis. Int J Numer Anal Methods Geomech. 2002;26(9):845-871. https://doi.org/10.1002/nag.224

42. Barthélémy JF, Dormieux L, Maghous S. Micromechanical approach to the modelling of compaction at large strains. Comput Geotech. 2003;30(4):321-338. https://doi.org/10.1016/S0266-352X(03)00006-5

43. Deudé V, Dormieux L, Maghous S, Barthélémy JF, Bernaud D. Compaction process in sedimentary basins: the role of stiffness increase and hardening induced by large plastic strains. Int J Numer Anal Methods Geomech. 2004;28(13):1279-1303. https://doi.org/10.1002/ nag. 387

44. Bernaud D, Dormieux L, Maghous S. A constitutive and numerical model for mechanical compaction in sedimentary basins. Comput Geotech. 2006;33(6-7):316-329. https://doi.org/10.1016/j.compgeo.2006.05.004

45. Houseknecht DW. Assessing the relative importance of compaction processes and cementation to reduction of porosity in sandstones. Am Assoc Pet Geol Bull. 1987;71(6):633-642. https://doi.org/10.1306/9488787f-1704-11d7-8645000102c1865d

46. Zhou Y, Rajapakse RKND, Graham J. A coupled thermoporoelastic model with thermo-osmosis and thermal-filtration. Int J Solids Struct. 1998;35(34-35):4659-4683. https://doi.org/10.1016/S0020-7683(98)00089-4

47. Yang Y, Guerlebeck K, Schanz T. Thermo-osmosis effect in saturated porous medium. Transp Porous Media. 2014;104(2):253-271. https://doi.org/10.1007/s11242-014-0332-5

48. Salomoni VA. A mathematical framework for modelling 3D coupled THM phenomena within saturated porous media undergoing finite strains. Compos Part B Eng. 2018;146:42-48. https://doi.org/10.1016/j.compositesb.2018.03.038

49. Karrech A, Poulet T, Regenauer-Lieb K. Poromechanics of saturated media based on the logarithmic finite strain. Mech Mater. 2012;51: 118-136. https://doi.org/10.1016/j.mechmat.2012.03.011

50. Lewis TW, Pivonka P, Smith DW. Theoretical investigation of the effects of consolidation on contaminant transport through clay barriers. Int J Numer Anal Methods Geomech. 2009;33(1):95-116. https://doi.org/10.1002/nag.708

51. Brüch A, Maghous S, Ribeiro FLB, Dormieux L. A constitutive model for mechanical and chemo-mechanical compaction in sedimentary basins and finite element analysis. Int J Numer Anal Methods Geomech. 2016;40(16):2238-2270. https://doi.org/10.1002/nag.2530

52. Brüch A, Guy N, Maghous S. Overpressure development in sedimentary basins induced by chemo-mechanical compaction of sandstones. Mar Pet Geol. 2019;104(October 2018):217-230. https://doi.org/10.1016/j.marpetgeo.2019.03.025

53. Maghous S, Dormieux L, Barthélémy JF. Micromechanical approach to the strength properties of frictional geomaterials. Eur J Mech A/Solids. 2009;28(1):179-188. https://doi.org/10.1016/j.euromechsol.2008.03.002

54. Zaoui A. Continuum micromechanics: survey. J Eng Mech. 2002;128(8):808-816. https://doi.org/10.1061/(ASCE)0733-9399(2002)128: $8(808)$

55. Dormieux L, Kondo D, Ulm FJ. Microporomechanics. John Wiley \& Sons L; 2006.

56. Charlez PA, Fairhurst C. Rock Mechanics. Petroleum Applications. 2 Paris: Editions Technip; 1997.

57. Perzyna P. Fundamental problems in viscoplasticity. Adv Appl Mech. 1966;243-277. https://doi.org/10.1016/S0065-2156(08)70009-7

58. Schneider F, Hay S. Compaction model for quartzose sandstones application to the Garn Formation, Haltenbanken, Mid-Norwegian Continental Shelf. Mar Pet Geol. 2001;18(7):833-848. https://doi.org/10.1016/S0264-8172(01)00032-0

59. Simo JC, Hughes TJR. Computational Inelasticity. New York: Springer-Verlag; 1998.

How to cite this article: Lemos PSB, Brüch A, Maghous S. Formulation of reference solutions for compaction process in sedimentary basins. Int J Numer Anal Methods Geomech. 2020;44:2135-2166. https://doi.org/10.1002/

nag.3129 